\title{
Phase-Locked Responses to Tones of Chinchilla Auditory Nerve Fibers: Implications for Apical Cochlear Mechanics
}

\author{
Andrei N. Temchin ${ }^{1}$ and Mario A. Ruggero ${ }^{1}$ \\ ${ }^{1}$ Hugh Knowles Center (Department of Communication Sciences and Disorders), Northwestern University, 2240 Campus Drive, \\ Evanston, IL 60208-3550, USA
}

Received: 16 July 2009; Accepted: 25 October 2009; Online publication: 17 November 2009

\begin{abstract}
Responses to tones with frequency $\leq 5 \mathrm{kHz}$ were recorded from auditory nerve fibers (ANFs) of anesthetized chinchillas. With increasing stimulus level, discharge rate-frequency functions shift toward higher and lower frequencies, respectively, for ANFs with characteristic frequencies (CFs) lower and higher than $\sim 0.9 \mathrm{kHz}$. With increasing frequency separation from $\mathrm{CF}$, rate-level functions are less steep and/or saturate at lower rates than at CF, indicating a CF-specific nonlinearity. The strength of phase locking has lower high-frequency cutoffs for $\mathrm{CFs}>4 \mathrm{kHz}$ than for $\mathrm{CFs}<$ $3 \mathrm{kHz}$. Phase-frequency functions of ANFs with CFs lower and higher than $\sim 0.9 \mathrm{kHz}$ have inflections, respectively, at frequencies higher and lower than CF. For $\mathrm{CFs}>2 \mathrm{kHz}$, the inflections coincide with the tiptail transitions of threshold tuning curves. ANF responses to $\mathrm{CF}$ tones exhibit cumulative phase lags of 1.5 periods for CFs $0.7-3 \mathrm{kHz}$ and lesser amounts for lower CFs. With increases of stimulus level, responses increasingly lag (lead) lower-level responses at frequencies lower (higher) than CF, so that group delays are maximal at, or slightly above, CF. The CF-specific magnitude and phase nonlinearities of ANFs with $\mathrm{CFs}<2.5 \mathrm{kHz}$ span their entire response bandwidths. Several properties of ANFs undergo sharp transitions in the cochlear region with CFs $2-5 \mathrm{kHz}$. Overall, the responses of chinchilla ANFs resemble those in other mammalian species but contrast with available measurements of apical cochlear vibrations in chinchilla, implying that either the latter are flawed or that a
\end{abstract}

Correspondence to: Mario A. Ruggero - Hugh Knowles Center (Department of Communication Sciences and Disorders) - Northwestern University - 2240 Campus Drive, Evanston, IL 60208-3550, USA. Telephone: +1-847-4913180; fax: +1-847-4912523; email: mruggero@ northwestern.edu nonlinear "second filter" is interposed between vibrations and ANF excitation.

Keywords: basilar membrane, cochlear apex, phase-frequency functions, rate-frequency functions

\section{INTRODUCTION}

Here, we describe the responses to tones with frequency $\leq 5 \mathrm{kHz}$ of chinchilla auditory nerve fibers (ANFs). This investigation was motivated by the dearth and conflictive nature of data on vibrations and their neural encoding in cochlear regions with low characteristic frequency $(\mathrm{CF})$. Available data show drastic differences between apical vibrations in guinea pig and chinchilla (the only species in which they have been measured in vivo) and, in the case of chinchilla, appear to be inconsistent with responses of low-CF ANFs in other mammalian species. In addition, possible discrepancies may exist between responses of low-CF ANFs in chinchilla vis-à-vis other species.

Accounts of vibrations at apical sites of the cochleae of live guinea pigs (Cooper 2006; Dong and Cooper 2006; Zinn et al. 2000) and chinchillas (Cooper and Rhode 1996; Rhode and Cooper 1996) are inconsistent, differing in essential details including the nature of nonlinearity (compressive in chinchilla (Rhode and Cooper 1996) but expansive in guinea pig, according to Zinn et al. (2000)) and its relationship to frequency tuning (i.e., level dependent in guinea pig (Zinn et al. 2000) but independent of level in chinchilla (Rhode and Cooper 1996)). The data for chinchilla (Cooper and Rhode 1996; Rhode and Cooper 1996) are probably not representative of those in intact cochleae because they were obtained after puncturing Reissner's 
membrane, a procedure that elevates low-frequency compound action potential (CAP) thresholds (Dong and Cooper 2006). Even in studies in which vibrations were measured without rupturing Reissner's membrane and CAP thresholds were not elevated (in guinea pig; Dong and Cooper 2006; Zinn et al. 2000), dysfunction may have gone undetected since lowfrequency CAP thresholds are insensitive to apical cochlear damage (Cooper and Rhode 1995; Harris 1979; Johnstone et al. 1979; Zinn et al. 2000).

The frequency tuning of vibrations at apical sites of the chinchilla cochlea is independent of stimulus level (Rhode and Cooper 1996), in contrast with the level dependence of rate-frequency functions of ANFs in squirrel monkey (Anderson et al. 1971; Rose et al. 1971). With increasing stimulus level, apical vibrations exhibit phase leads and lags, respectively, for frequencies lower and higher than CF (Rhode and Cooper 1996). Such a pattern is the reverse of that in responses of low-CF ANFs (Anderson et al. 1971; Palmer and Shackleton 2009; Rose et al. 1971; van der Heijden and Joris 2003; Carney and Yin 1988) and inner hair cells (IHCs; Cheatham and Dallos 2001; Dallos 1986) in other species and also the reverse of the patterns of basilar membrane (BM) vibrations at basal sites in chinchilla (Ruggero et al. 1997) as well as other species (Cooper and Rhode 1992; Nuttall and Dolan 1993; Ren and Nuttall 2001; Rhode 1971). Additionally, the level dependence of phases in responses to tones of low-CF chinchilla ANFs (Rhode and Cooper 1997) may differ from the level dependence in responses to various stimuli in chinchilla (RecioSpinoso et al. 2005) and other species (Anderson et al. 1971; Palmer and Shackleton 2009; van der Heijden and Joris 2003; Carney and Yin 1988).

Since genuine species differences might account for the discrepancies and inconsistencies, it was deemed important to ascertain whether the responses to tones of low-CF ANFs in chinchilla resemble those in other mammalian species. The present paper shows that that is the case and that important differences exist between the responses of low-CF ANFs and available mechanical recordings from apical sites of the chinchilla cochlea. Preliminary results of this investigation were published in abstract form (Temchin and Ruggero 2000, 2001).

\section{METHODS}

\section{Preparations for single-unit recordings}

The experimental techniques employed here have been described elsewhere (Ruggero et al. 1996; Ruggero and Rich 1983, 1987). All animal procedures were approved by the Animal Care and Use Committee of Northwestern University. Adult chinchillas were initially anesthetized with ketamine hydrochloride
(100 mg/kg, injected subcutaneously) and diallylbarbituric acid in urethane $(1 \mathrm{~g} / \mathrm{kg}$, injected intraperitoneally). Deep anesthesia was maintained with supplemental doses of the latter anesthetic to completely eliminate limb withdrawal reflexes (at the end of the experiments, the animal was killed by decapitation while still deeply anesthetized). Core body temperature was kept near $38^{\circ} \mathrm{C}$ by means of a servocontrolled electrical heating pad. A polyethylene tube inserted into the trachea facilitated unassisted respiration. The pinna was resected and part of the bony external ear canal was chipped away to visualize the umbo of the tympanic membrane. The tip of the earphone-coupling speculum was then placed against and sealed to the bony rim of the ear canal. The tendon of the tensor tympani muscle was severed. All experimental recordings were carried out in a soundattenuated booth in chinchillas whose heads were firmly immobilized. CAPs were recorded with a silver ball electrode placed on the round window in advance of single-unit recordings from the auditory nerve. These were discontinued if CAP thresholds $(0.5$ to $16 \mathrm{kHz}$ in half-octave steps) became elevated by more than $6 \mathrm{~dB}$.

\section{Single-unit recordings}

The auditory nerve was approached superiorly after craniotomy and partial aspiration of the lateral cerebellum. Capillary-glass microelectrodes (filled with $3 \mathrm{M}$ $\mathrm{NaCl}$ solution, impedance 20-80 $\mathrm{M} \Omega$ ) were initially positioned under observation with an operation microscope and were advanced into the nerve by means of a remotely controlled hydraulic micropositioner (Kopf 650 ). The microelectrode signal was amplified (Dagan), filtered $(0.3-3 \mathrm{kHz})$ by means of a "spike conditioner" (PC1, Tucker-Davis Technologies (TDT)), and displayed on an oscilloscope. A discriminator (SD1, TDT) triggered standard pulses early on the rising edge of the spikes just above background noise. The discriminator level was continuously monitored and adjusted to maintain faithful triggering. Correct computation of responses phases, a central aim of the present work, depends crucially not only on accurate phase calibration of the acoustic signal but also on taking into account delays involved in spike timing (due to amplification and filtering). Those delays, amounting to $120 \mu \mathrm{s}$, were carefully measured and compensated online. The pulse times were stored in digital media,

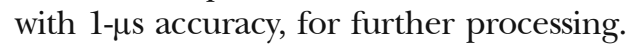

Acoustic stimuli were generated by means of 16-bit TDT equipment. Sinusoidal digital waveforms were converted into analog electrical signals by means of a D/A converter (TDT DA3-2; sampling rate $100 \mathrm{kHz}$ ), low-pass filtered ( $24 \mathrm{kHz}$; Kemo), and transduced into acoustic waveforms by a Beyer DT-48 earphone. The amplitudes and phases of acoustic stimuli, measured 
at the beginning of each experimental session with a miniature microphone with its tip located within $2 \mathrm{~mm}$ of the eardrum, were recorded digitally in a calibration table. The table allowed computer-aided specification of the sound pressure level (SPL; re $20 \mu \mathrm{Pa}$ ) and the phase of acoustic sinusoids. The spectral composition of low frequency stimuli produced by the sound system has been described in some detail elsewhere (see Table 1 in Ruggero et al. 1996). Briefly, for low-frequency tones (below $1 \mathrm{kHz}$ ), the magnitude of second-harmonic distortion measured in an artificial cavity was typically less than $-50 \mathrm{~dB}$ re the fundamental at stimulus levels of 106$108 \mathrm{~dB}$, but in live chinchillas, it could reach $-28 \mathrm{~dB}$ at $100 \mathrm{~Hz}$ (Ruggero et al. 1996; see also Kim et al. 1980). For higher frequencies, the magnitude of second-harmonic distortion did not exceed $-55 \mathrm{~dB}$.

\section{Stimulus protocol}

White noise bursts of 50-ms duration (presented 3/s) were used as search stimuli. Upon isolation of each ANF, a 10-s sample of spontaneous activity (SR) was obtained. Then, a frequency-threshold tuning curve (FTC) was recorded using an automated adaptive algorithm (Kiang et al. 1970; see also Liberman and Kiang 1978; Temchin et al. 2008b). After smoothing each FTC (using threepoint averaging), a third-order polynomial equation was fitted to the FTC tip within $15 \mathrm{~dB}$ of its minimum. CF and $\mathrm{CF}$ thresholds were defined as the frequency and level of the fit minimum (see Temchin et al. 2008b).

The bulk of the data consists of responses to five or ten repetitions of 100-ms tones presented every $300 \mathrm{~ms}$. Tones with 5-ms onsets and offsets (shaped as $\cos ^{2}$ functions) were presented as series in which level was kept constant while frequency was varied from $50 \mathrm{~Hz}$ up to $5 \mathrm{kHz}$ in steps of $10-100 \mathrm{~Hz}$, depending on CF. Frequency steps were always small enough so that the phases of responses for adjacent stimulus frequencies did not differ by more than a quarter of a cycle, thus ensuring that phases could be correctly unwrapped (see Figs. 1C and 9). Tones were first presented at $70 \mathrm{~dB}$ SPL, which elicit high firing rates at frequencies around CF but almost never "peak splitting" or related phenomena (Fig. 1B; Ruggero et al. 1996). Subsequently, tones were presented at threshold and other stimulus levels, as many as possible, in no particular order. Overall, the database includes 1,791 rate-frequency series recorded from 615 ANFs in 63 chinchillas.

\section{Data analyses}

Average response rate, mean phase, its standard deviation, and vector strength (VS; a measure of the strength of phase locking; Goldberg and Brown 1969) were computed from the times of the spikes elicited by tones presented at each frequency-level combination. Spike times were also used to construct period histograms, such as those illustrated in Figure 1A for responses of a single ANF to tones presented at $80 \mathrm{~dB}$ SPL. VS was computed on the basis of individual spike times (Goldberg and Brown 1969). VS values were considered reliable if $N$ (number of spikes) $\geq 32$. Phase values were considered reliable if the standard deviation of the phases $<0.375$ periods and $N \geq 40$. The standard deviation $(\sigma)$ is given by Eq. 1 (Littlefield 1973):

$$
\sigma(\text { periods })=[(0.5 * V S) * \sqrt{2}] / N
$$

With $\sigma<0.375$ periods, VS $>0.298$ and $2 * N^{*} V S^{2}$ (the "Rayleigh number") $>7.1$. With $2 * N * V S^{2}=7.1$, phase locking is significant with $p<0.05$ (Mardia and Jupp 2000). These criteria for data selection are less restrictive than in previous comparable studies, e.g., $\sigma<0.07$ periods and $N>600$ spikes in Pfeiffer and Kim (1975) or $2^{*} N^{*} V S^{2}=10,10.6$, and 13.8 , respectively, in Ruggero et al. (1996), Temchin et al. (2005), and Buunen and Rhode (1978). Nevertheless, test-retest differences between average phases for data considered reliable were frequency independent and small, $7^{\circ} \pm 5^{\circ}(\operatorname{mean} \pm \sigma ; N=170)$ for frequencies $\leq 1 \mathrm{kHz}$, and $15^{\circ} \pm 10^{\circ}(N=31)$ for frequencies $2-3.5 \mathrm{kHz}$.

The generic and CF-independent rate-level function used to generate the predicted rate-frequency functions of Figure 4D, F (illustrated in all panels of Fig. 5, thick solid lines) was of the form expressed in Eqs. 1 and 2 of Sachs et al. (1989), with a single modification: In Eq. 2, the exponent "1.77" was replaced by "1.6". Variables: $\theta_{i}=$ pressure (Pascals) corresponding to $15 \mathrm{~dB}$ re threshold; $\theta_{\mathrm{E}}=$ pressure (Pascals) corresponding to $11 \mathrm{~dB}$ re threshold; $R_{\mathrm{M}}=1$. These parameters were obtained by fitting the modified equations of Sachs et al. (1989) to the average of rate-level functions at $\mathrm{CF}$ for several representative ANFs with $\mathrm{CFs}<3 \mathrm{kHz}$.

The phases of chinchilla ANF responses to tones with frequency well below CF do not change appreciably from below rate threshold up to 80-90 dB SPL (Fig. 1B). At such levels, however, a sharp phase shift occurs, which may be accompanied by "peak splitting" and a sharp notch in rate-intensity functions (Ruggero et al. 1996). Therefore, unless otherwise specified, all phases reported here correspond to responses evoked by tones presented at $\leq 80 \mathrm{~dB}$ SPL, with period histograms that did not exhibit "peak splitting". Individual rate-, vector strength-, and phasefrequency functions were smoothed by three-point averaging (except in Figs. 1B, C). Phases were corrected off line for neural and synaptic delays (1 ms) to yield estimates of the mechanical input to the IHCs (Ruggero et al. 1996). 

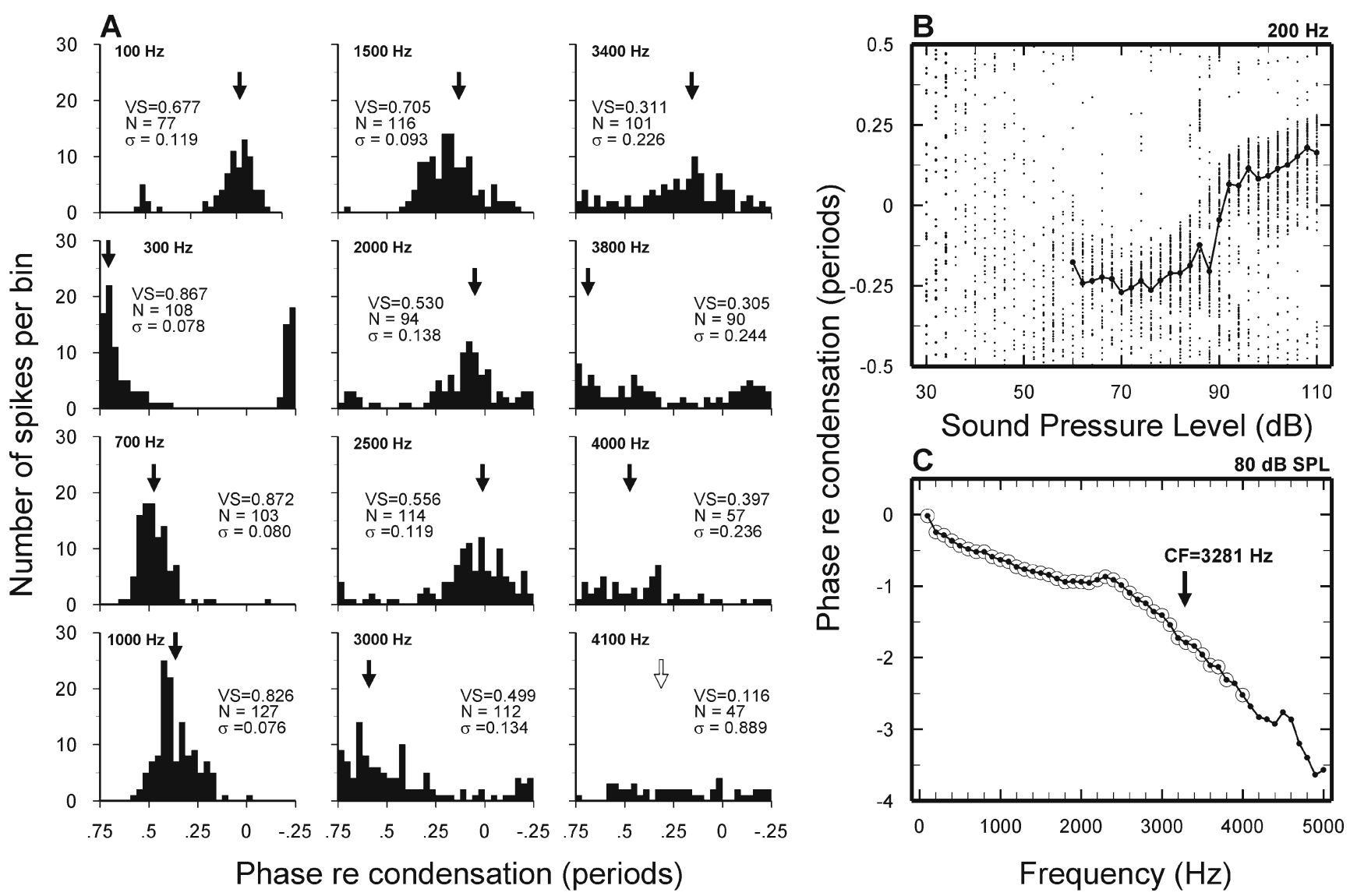

FIG. 1. Period histograms and phases of ANF responses to tones. A Representative 32-bin period histograms of responses of an ANF (CF= $3.3 \mathrm{kHz}$ ) to tones presented at $80 \mathrm{~dB}$ SPL. Vertical arrows indicate mean response phase. Vector strength (VS), number of spikes $(N)$, and standard deviation of the phase $(\sigma)$ are indicated. VS was computed on the basis of individual spikes times rather than from binned data. B Phase-level scatter plot for responses to $200-\mathrm{Hz}$ tones. Stimulus levels were varied in random order, with 2-dB steps, between 30 and

Occasionally, ANF data were recorded as responses to series of constant-frequency tones presented with stimulus levels varied randomly, in 2-dB steps, from below rate threshold up to the limits imposed by the hardware. Such responses were used to construct phase-level scatter plots, such as the one illustrated in Figure 1B (see Kiang et al. 1970; Liberman and Kiang 1984; Ruggero et al. 1996; Temchin et al. 1997).

\section{RESULTS}

\section{Asymmetry of ANF rate-frequency functions}

Figure 2 presents families of iso-SPL rate-frequency curves for representative ANFs with CFs spanning most of the phase-locking range. At near-threshold stimulus levels, rate-frequency curves have relatively narrow peaks at frequencies (best frequencies, BFs) close to $\mathrm{CF}$ and are fairly symmetrical (we distinguish between $\mathrm{BF}$ and $\mathrm{CF}$. BF is the stimulus frequency
$110 \mathrm{~dB}$ SPL. Spike times (dots) are displayed modulo one period. Connected symbols represent average response phases. Note abrupt phase shift between 88 and $92 \mathrm{~dB}$ SPL. C Phases (dots) of responses to 80-dB SPL tones, including those represented in A, plotted against frequency. Frequency step, $100 \mathrm{~Hz}$. Open circles indicate phases for responses that satisfied a reliability criterion (see "Methods" section). Responses for frequencies $\geq 4.1 \mathrm{kHz}$ did not meet the criterion (see also A). All data were corrected for 1-ms neural/synaptic delay.

producing the largest response, without regard to stimulus level or cochlear health or development. CF is the $\mathrm{BF}$ of responses at threshold in mature and healthy cochleae; see Fig. 13 of Robles and Ruggero 2001). With increases in stimulus level, rate-frequency functions broaden, as the upper and lower cutoff frequencies increasingly diverge from CF. For CFs $<900 \mathrm{~Hz}$ (panels A-C), increases in stimulus level cause rate-frequency curves to broaden preponderantly toward frequencies higher than CF. For CFs $\approx$ $900 \mathrm{~Hz}$ (panel D), rate-frequency curves are nearly symmetrical at all levels. For $\mathrm{CFs}>1,000 \mathrm{~Hz}$ (panels E$\mathrm{H})$, rate-frequency curves broaden preponderantly toward frequencies $<\mathrm{CF}$.

The level-dependent shifts of rate-frequency curves were quantified by computing their "centers of gravity", the frequencies which partition the areas under the rate-frequency curves into equal halves (i.e., areas are identical on either side of the centers of gravity). The centers of gravity of rate-frequency 


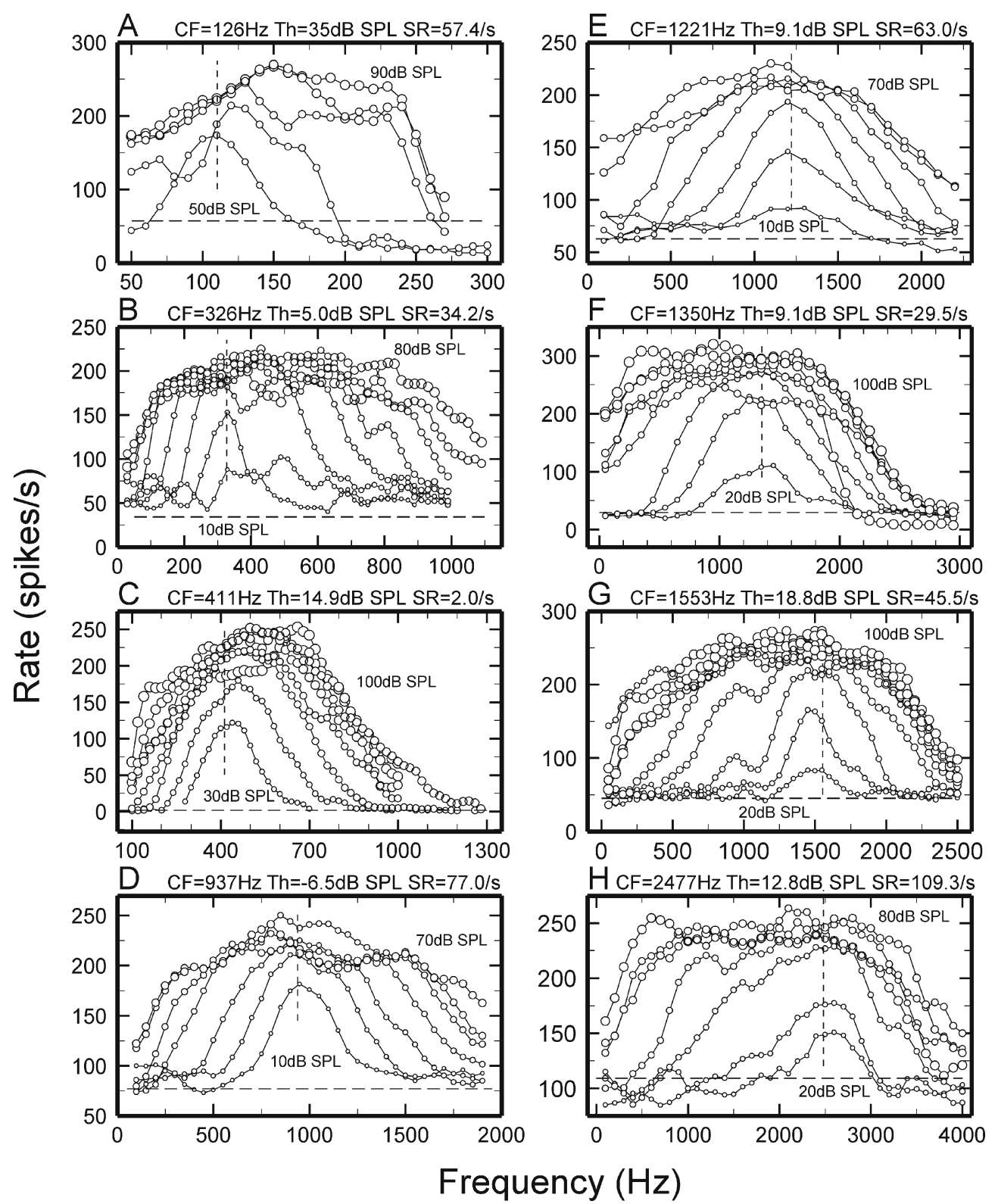

FIG. 2. Rate-frequency functions for ANF responses to tones. Each panel presents data from a single ANF with CF, CF threshold, and SR indicated at the top. SPL is the parameter. Symbol sizes are proportional to stimulus level, which ranged up to 70-100 dB SPL,

curves measured at $70 \mathrm{~dB}$ SPL, expressed in octaves re $\mathrm{CF}$, are plotted in Figure 3 against $\mathrm{CF}$ (open gray circles; left scale). Negative values (plotted upward) and positive values (plotted downward) indicate, respectively, level-dependent shifts toward frequencies lower and higher than CF. The dashed line, a leastsquares fit that accounts for $77 \%$ of the variance of the centers of gravity, indicates that, on average, shifts change polarity at CFs around $870 \mathrm{~Hz}$.

Except for CFs near $0.9 \mathrm{kHz}$, the FTCs of chinchilla ANFs are asymmetrical in shape (Fig. 4A, D; see Temchin et al. 2008b). For $\mathrm{CFs}<0.9 \mathrm{kHz}$ (Fig. 4A), partial bandwidths (i.e., bandwidths measured in 10-dB steps. The lowest and highest stimulus SPLs are indicated. Horizontal dashed lines indicate SR. Vertical dashed lines mark CF (obtained from FTC; see "Methods" section).

between CF and either the lower or upper FTC limbs; graphically defined in Fig. 4A, B) are larger for frequencies $>\mathrm{CF}\left(\mathrm{BW}_{\mathrm{a}}\right)$ than for frequencies $<\mathrm{CF}$ $\left(\mathrm{BW}_{\mathrm{b}}\right)$. The reverse is true for $\mathrm{CFs}>0.9 \mathrm{kHz}$ (Fig. 4D). Figure 3 includes trend lines (connected blue symbols, right scale) for the variation of a measure of FTC asymmetry as a function of the CF of chinchilla ANFs (Temchin et al. 2008b). Perfect symmetry is indicated by a value of one (horizontal dashed line and blue right scale). Values $<1$ indicate that $\mathrm{BW}_{\mathrm{a}}>\mathrm{BW}_{\mathrm{b}}$ (e.g., Fig. 4A). Values $>1$ indicate the reverse (e.g., Fig. 4D). Figure 3 demonstrates that FTC asymmetry and the level-dependent shifts of the center of gravity re $\mathrm{CF}$ 


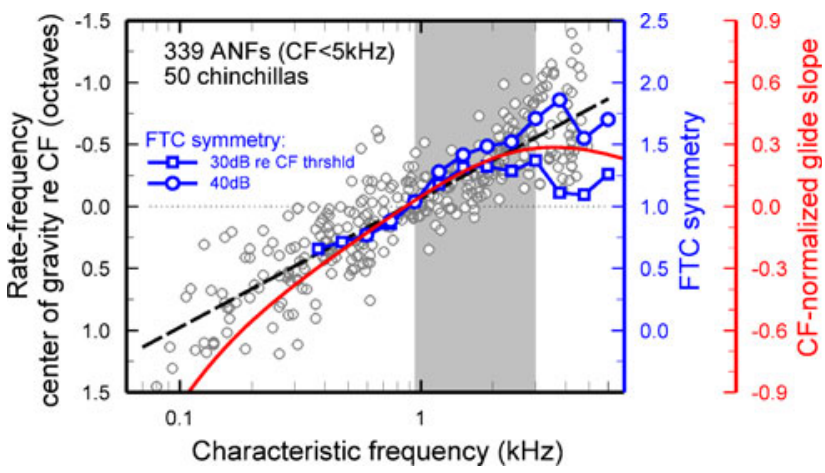

FIG. 3. Variation with CF of level-dependent frequency shifts of rate-frequency curves, asymmetries of FTCs, and impulse response frequency glides. Level-dependent frequency shifts (open gray symbols) were estimated by dividing the center frequencies of the rate-frequency curves (i.e., their centers of gravity) measured at $70 \mathrm{~dB}$ SPL by the CF. Positive and negative values, expressed as octaves re $\mathrm{CF}$, indicate shifts toward higher and lower frequencies, respectively, as a function of increasing SPL. The dashed line is a fit to the frequency-shift data. The connected blue symbols indicate trend lines for the asymmetry ratios of ANF FTCs; data from Figure 5 of Temchin et al. (2008b) in chinchilla. An FTC symmetry ratio (right scale) $<1$ indicates that the partial bandwidth above CF $\left(\mathrm{BW}_{\mathrm{a}}\right.$ of Figs. 4A, D) is wider than the partial bandwidth below CF $\left(B W_{b}\right.$ of Figs. 4A, D). Symmetry ratios $>1$ signify the reverse relation. The red trace indicates the trend of the CF-normalized slopes of instantaneous frequencies at the onset of Wiener kernels of chinchilla ANF responses to noise and BM responses to clicks; data from Figure 14 of Temchin et al. (2005). Gray shading indicates the CF region within which phase-frequency curves undergo a change in curvature, from "concave-upward" to "concave-downward"; see Figure 11.

flip direction at the same CF, about $900 \mathrm{~Hz}$. It is shown below that this coincidence is not fortuitous.

\section{Prediction of rate-frequency functions from FTCs}

If cochlear vibrations were either linear or exhibited (only) frequency-independent nonlinearities and given that for any single ANF, the translation of IHC receptor potentials into response rates is independent of stimulus frequency (Zagaeski et al. 1994), ratefrequency functions (Fig. 4B, E) should be predictable from FTCs (Fig. 4A, D). Rate-frequency curves (Figs. 4C, F) were predicted using a "generic" ratelevel curve. The generic curve (thick lines in Fig. 5) was obtained by fitting a standard equation (adapted from Sachs et al. 1989; see "Methods" section) to the average of rate-level curves at CF for several representative ANFs. Used in combination with a given FTC, the generic rate-level function yields ratefrequency curves (e.g., Fig. 4C, F) that qualitatively resemble the measured ones (Fig. 4B, E), including the directions of their frequency shifts with increased stimulus level. In other words, the asymmetric leveldependent shifts of the rate-frequency functions are necessary consequences of the asymmetries of the FTCs.

\section{CF-specific variation of ANF rate-level curves}

The measured (panels $\mathrm{B}$ and $\mathrm{E}$ ) and predicted ( $\mathrm{C}$ and F), rate-frequency curves of Figure 4 are similar but not identical, differing especially at high stimulus levels. In particular, the bandwidths of the measured curves are narrower than those of the predicted curves, indicating that responses rates are limited by CF-specific effects. To explore these effects, Figure 5 presents driven rates (i.e., average rate minus SR) plotted against threshold-normalized stimulus levels (i.e., SPL minus FTC threshold). Rate-level functions for frequencies lower and higher than $\mathrm{CF}$ are shown in the left $(\mathrm{A}-\mathrm{D})$ and right $(\mathrm{E}-\mathrm{H})$ panels, respectively. The data of Figure 5 indicate that the slopes of ratelevel curves for ANF responses to tones are frequency dependent, varying systematically with frequency relative to CF: as stimulus frequencies depart from $\mathrm{CF}$, their slopes are lower than for $\mathrm{CF}$ tones. In other words, rates of growth are tuned to CF. In most ANFs, the CF-specific effects are the strongest for frequencies higher than $\mathrm{CF}$ (panels E, G, and $\mathrm{H}$ ), with ratelevel functions which often saturate at lower rates than at CF. In those ANFs, the CF-specific effects for frequencies $<\mathrm{CF}$ are milder than for frequencies $>\mathrm{CF}$ and saturation is rarely reached. However, reverse effects were found in a minority of ANFs (panels B and $\mathrm{F}$ ). The $\mathrm{CF}$ specificity of the rate-level slopes of low-CF ANFs probably indicates the presence of a CFspecific nonlinearity at the input to the IHC stereocilia, as is the case at the base of the cochlea, where the nonlinearity of vibrations of the $\mathrm{BM} /$ organ of Corti/tectorial membrane complex are CF specific (Rhode 2007; Rhode and Recio 2000; Ruggero et al. 1997).

\section{Variation of VS with CF and with stimulus frequency and level}

Each period histogram (e.g., Fig. 1A) consists of a DC component (the average rate) and an AC (or phaselocked) component, i.e., the periodic variation of the instantaneous rate around the average rate (Kim and Molnar 1979). Subtracting SR from the average rate yields the driven rate. The variations with stimulus level and frequency of the driven rate and the magnitude of the phase-locked component are shown in the top- and middle-row panels of Figure 6 for two ANFs with CFs of 0.4 and $2.5 \mathrm{kHz}$ (left- and rightcolumn panels). The CFs were selected to illustrate similarities and differences between the $\mathrm{AC}$ and $\mathrm{DC}$ response components as functions of CF. The bottom panels show VS, a measure of the strength of phase locking (Goldberg and Brown 1969) which varies between 0 (flat period histograms) and 1 (all spikes in the same bin). In the case of the ANF with $\mathrm{CF}=$ 

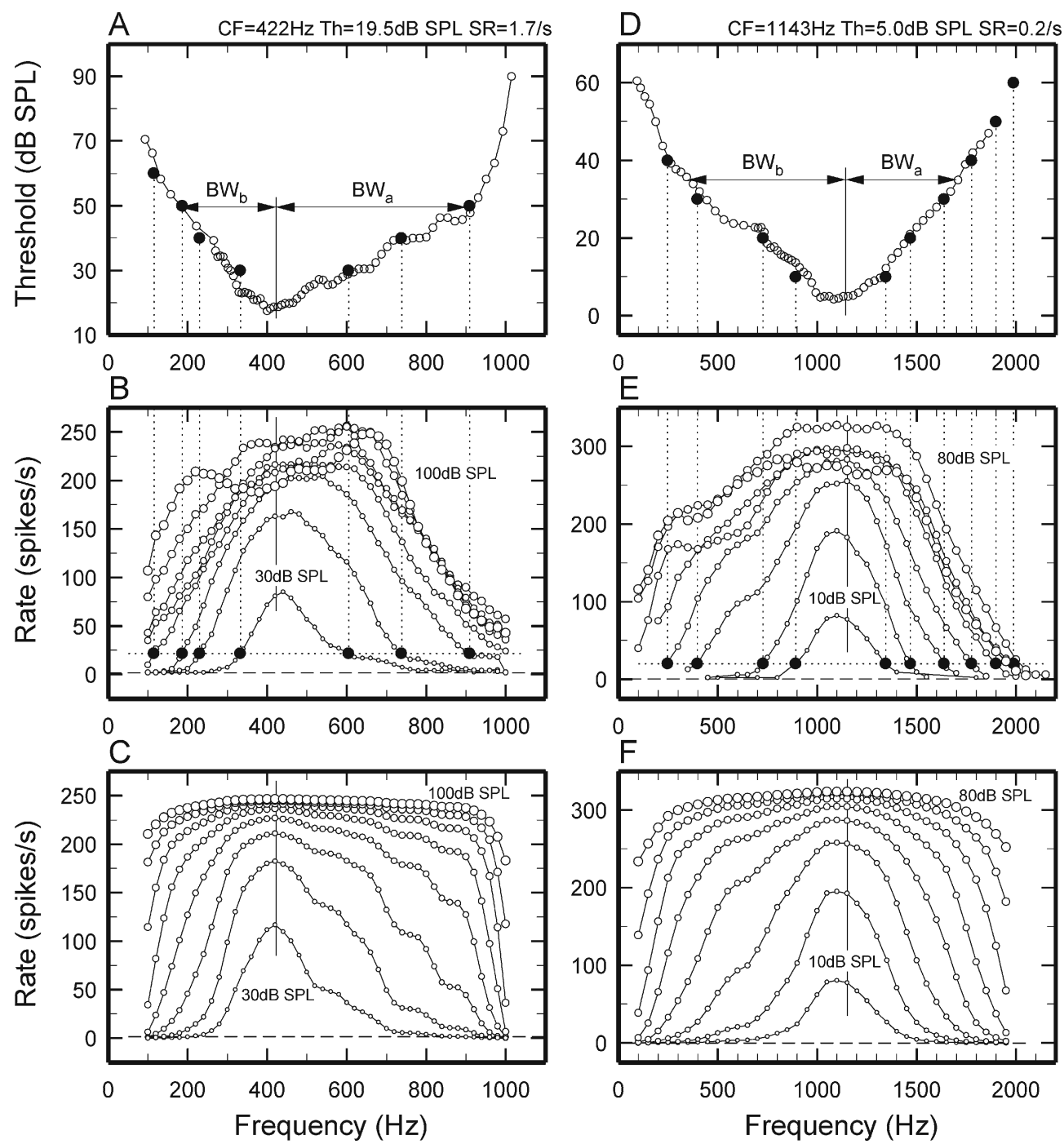

FIG. 4. Representative rate-frequency functions, measured and derived from FTCs, for ANFs with CFs lower and higher than $900 \mathrm{~Hz}$. Left column: data for an $\mathrm{ANF}$ with $\mathrm{CF}=422 \mathrm{~Hz}$. Right column: data for an ANF with $\mathrm{CF}=1,143 \mathrm{~Hz}$. Vertical solid lines mark CFs. Horizontal dashed lines in B, C, E, and F indicate SR. A, D FTCs measured with an adaptive procedure (see "Methods" section). B, E Measured rate-frequency functions. Filled circles mark frequencies at

$0.4 \mathrm{kHz}$, the driven rate and the $\mathrm{AC}$ component are similarly tuned because of the uniformly high values of VS, which are saturated even at rate threshold. In contrast, in the case of the ANF with $\mathrm{CF}=2.5 \mathrm{kHz}$, the driven rate and the AC component vary quite differently as functions of stimulus level and frequency because, as stimulus frequency increases, saturation of VS occurs at increasingly lower values.

Figure 7A shows the variation of VS with stimulus frequency for responses to tones presented at $70 \mathrm{~dB}$ SPL. The open circles and squares indicate average VS, computed in nonoverlapping 1/3-octave frequency bands, for $\mathrm{CFs}>4$ and $<3 \mathrm{kHz}$, respectively. At most stimulus frequencies (indicated by a dot

within the open squares), VSs for CFs $<3$ and $>4 \mathrm{kHz}$ are significantly different ( $p<0.0005$; Student's $t$ tests). For frequencies $<0.8 \mathrm{kHz}$, VS is larger for $\mathrm{CFs}>4 \mathrm{kHz}$ than for $\mathrm{CFs}<3 \mathrm{kHz}$. For higher frequencies, VS decays for the two $\mathrm{CF}$ groups at different rates and VS is larger for $\mathrm{CFs}<3 \mathrm{kHz}$ than for $\mathrm{CFs}>4 \mathrm{kHz}$. The corner frequency (the frequency at which VS equals 0.707 of the maximum) is $1.2 \mathrm{kHz}$ for $\mathrm{CFs}>4$ and $2.1 \mathrm{kHz}$ for $\mathrm{CFs}<3 \mathrm{kHz}$. Thus, the bandwidth of phase locking is about 0.75 octave wider for ANFs with $\mathrm{CFs}<$ $3 \mathrm{kHz}$ than for ANFs with CFs $>4 \mathrm{kHz}$. Figure 7A also allows for comparison of the VSs for ANFs with CFs $<$ $3 \mathrm{kHz}$ (filled squares) and $>4 \mathrm{kHz}$ (filled circles) for the subpopulation of ANFs with $\mathrm{SRs} \leq 1 \mathrm{spike} / \mathrm{s}$ CFs. 

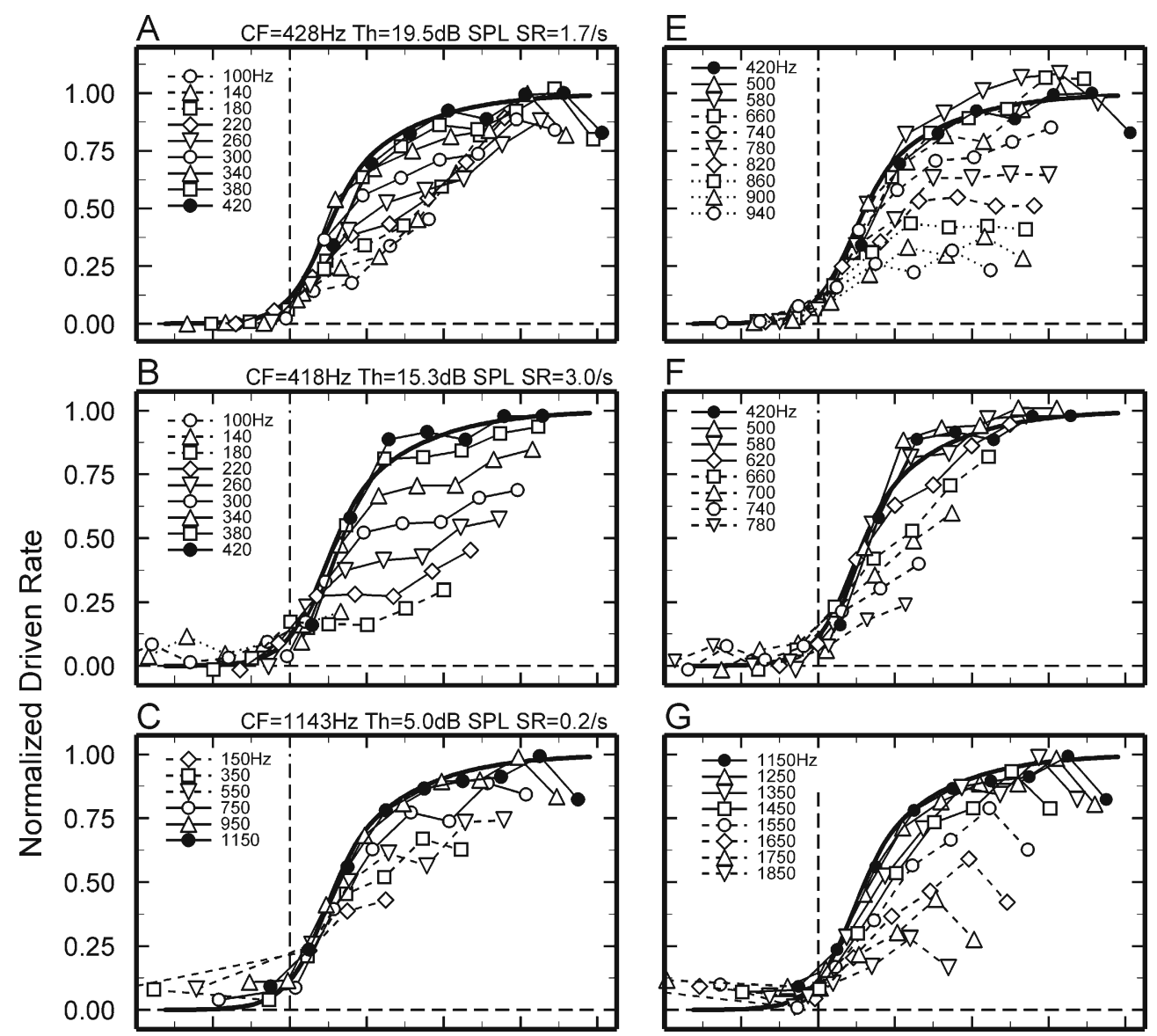

D $\quad \mathrm{CF}=1553 \mathrm{~Hz}$ Th=18.8dB SPL SR $=45.5 / \mathrm{s}$
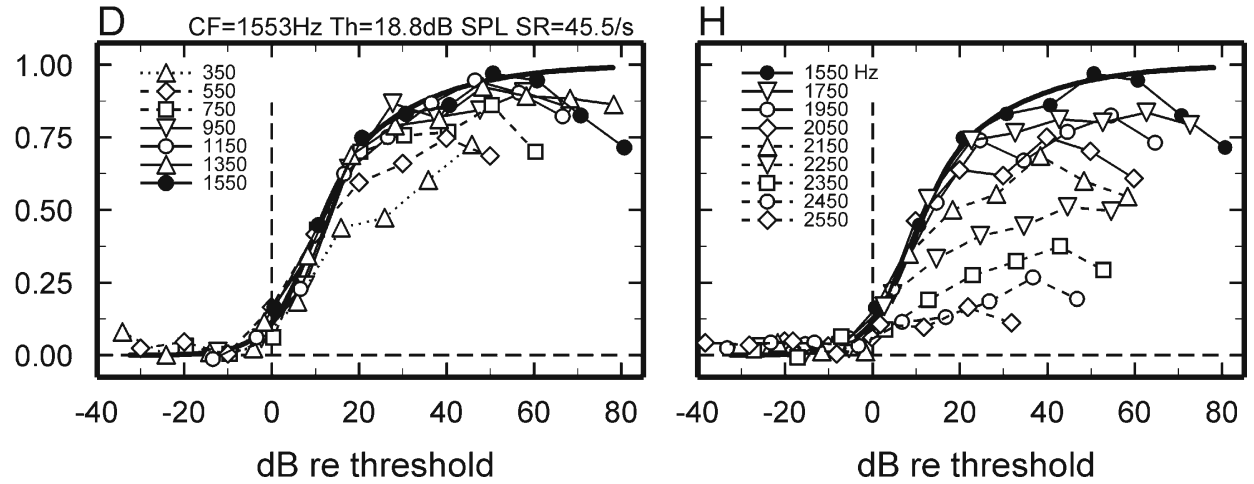

FIG. 5. Normalized driven rate-vs.-level functions. Ordinates: driven rates (i.e., response rates minus SR) normalized to driven rate at CF (thus, " 0 " = SR and " 1 " = maximal driven rate at CF). Abscissae: stimulus levels normalized to threshold for each stimulus frequency (i.e., stimulus SPL minus threshold SPL). Thus, " $0 \mathrm{~dB}^{\prime \prime}$ indicates threshold SPL at a given frequency. Legends indicate stimulus frequencies. Rates for the stimulus frequency closest to $\mathrm{CF}$

Bandwidths are larger by 0.4 octaves for $\mathrm{CFs}<3 \mathrm{kHz}$ than for $\mathrm{CFs}>4 \mathrm{kHz}$. In the case of ANFs with $\mathrm{CFs}>$ $4 \mathrm{kHz}$ (circles), VS differences between the low-SR subpopulation and the entire population are not statistically significant.

Figure $7 \mathrm{C}$ allows for comparison between the level of the $2.5-\mathrm{kHz}$ tones ( $70 \mathrm{~dB}$ SPL; horizontal line) and the levels (squares) at which responses to $2.5-\mathrm{kHz}$ are plotted as filled circles. Each row represents a different ANF. ANFs with CFs of 428 and $418 \mathrm{~Hz}$ were recorded in different animals. Thick lines indicate a generic (average) rate-level function (see Methods). Note that in some cases (E, F), maximal response rates occurred at frequencies other than $\mathrm{CF}$, indicating that $\mathrm{BF}$ shifted with stimulus level.

tones are presumably saturated, i.e., rate thresholds (circles) plus $10 \mathrm{~dB}$ (Johnson 1980; see also Pfeiffer and Kim 1975; Kim and Molnar 1979). The rate thresholds were computed from average FTCs for chinchilla ANFs; Fig. 4 of Temchin et al. 2008b. Figure $7 \mathrm{~B}$ plots VS against CF (open symbols) for responses to $2.5-\mathrm{kHz}$ tones. The VS values, presumably saturated in the $\mathrm{CF}$ range $1,625-6,525 \mathrm{~Hz}$ (gray area), 

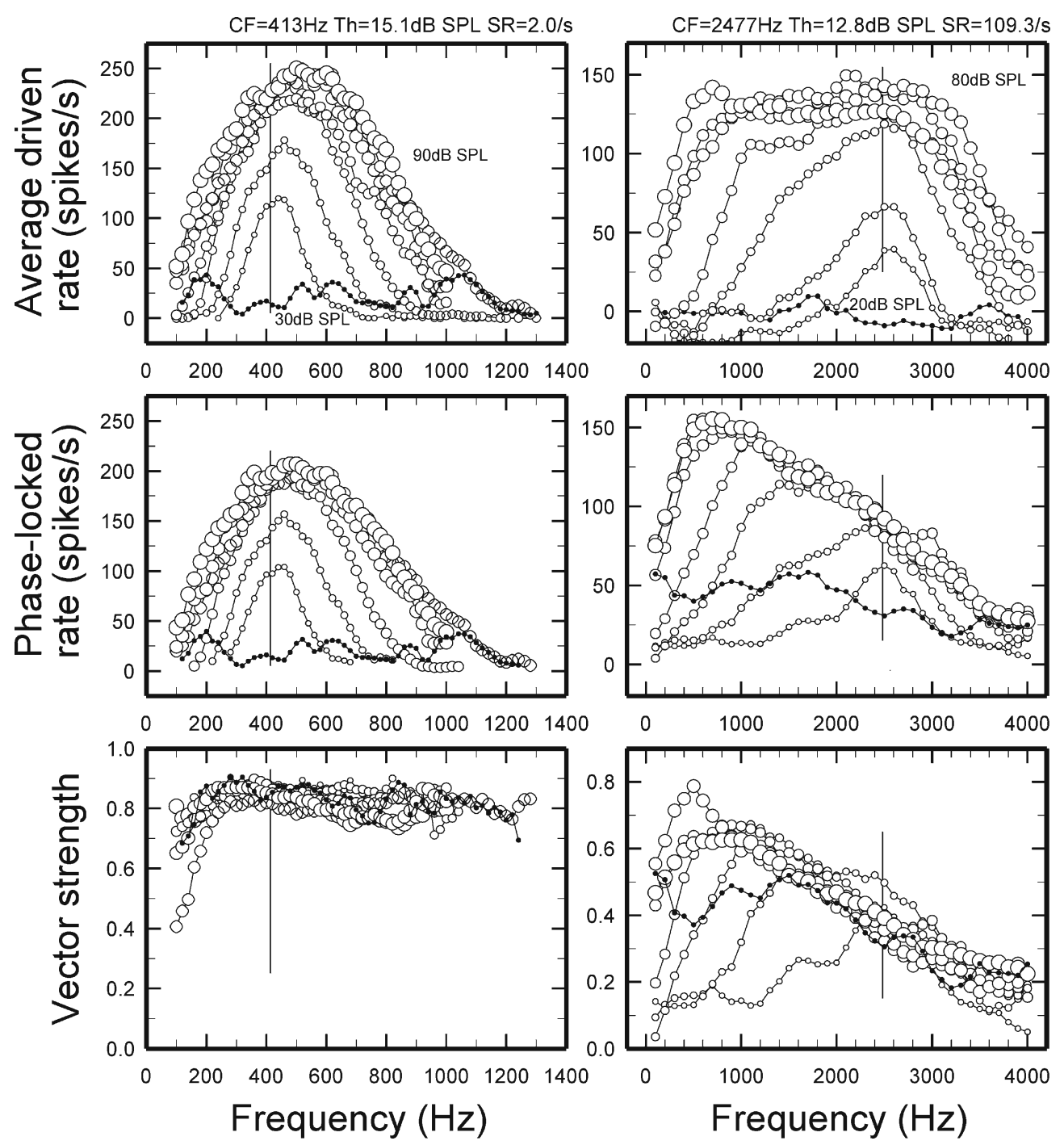

FIG. 6. The AC and DC components of ANF responses as functions of stimulus frequency and level. Response magnitudes are plotted against stimulus frequency, with stimulus SPL as the parameter, for the responses of ANFs with CFs of $0.4 \mathrm{kHz}$ (left) and $2.5 \mathrm{kHz}$ (right). Upper: DC component, i.e., average driven rate. Middle: the AC

are roughly constant for $\mathrm{CFs}<3 \mathrm{kHz}$ (circles), decrease abruptly between $\mathrm{CFs}$ of 3 and $4 \mathrm{kHz}$ (triangles), and then remain constant for $\mathrm{CFs}>$ $4 \mathrm{kHz}$ (squares). Linear fits for $\mathrm{CFs}<3$ and $>4 \mathrm{kHz}$ (horizontal lines) have slopes that do not differ significantly from zero. The mean VSs for CFs $<3$ and $>4 \mathrm{kHz}, 0.4$ and 0.2 , respectively (brackets: means \pm 1 standard deviation), are significantly different ( $p<0.0005$, Student's $t$ test). VS jumps in the 3-4$\mathrm{kHz}$ CF range were also observed for stimulus frequencies of $1.5,2$, and $3 \mathrm{kHz}$ (not shown), consistent with the interpretation that the VS jump of Figure $7 \mathrm{~B}$ does not reflect (gradual and tonotopic mechanical) changes in sensitivity to $2.5-\mathrm{kHz}$ stimuli (Fig. 7C) but rather a step-like change of the proper- component, i.e., amplitude of the fundamental Fourier component. Bottom: VS, i.e., AC component normalized to average rate. Symbol sizes vary monotonically with stimulus level. Filled circles represent data obtained at rate threshold. Vertical lines mark CF.

ties of IHCs and/or their synapses in the $3-4-\mathrm{kHz}$ CF region.

\section{Phase-frequency curves}

Figure 8 presents putative phases of peak IHC depolarization re condensation at the eardrum (deduced from the phases of ANF responses; see "Methods" section) for tone stimuli presented at $70 \mathrm{~dB}$ SPL. Each panel presents phase-frequency curves for CFs within a 1/3-octave band, with the center CFs indicated by dashed vertical lines. Phase unwrapping, i.e., the concatenations of phases into unique phase-frequency curves, was straightforward because data were collected as frequency series with small steps (see Figs. 1C and 9), 

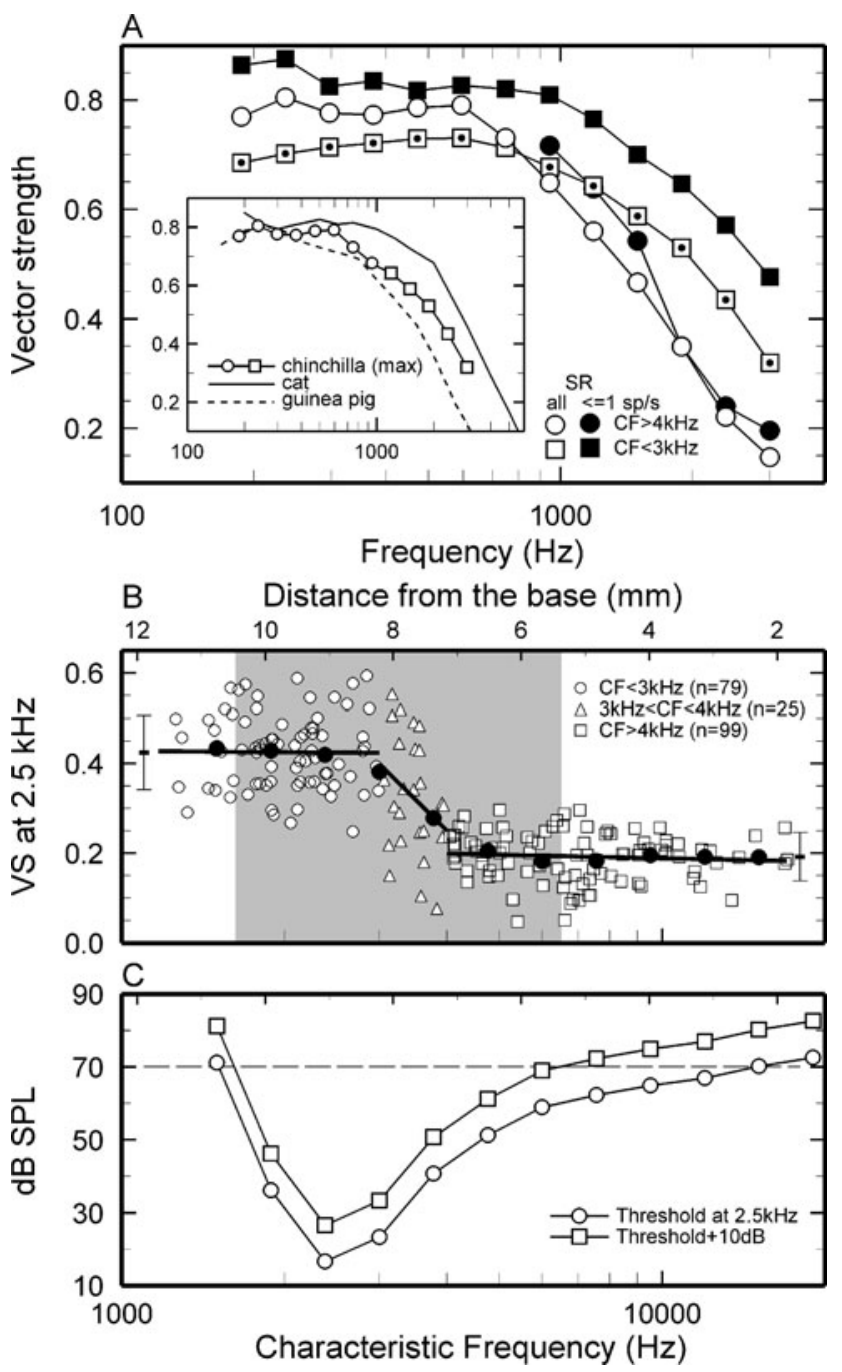

FIG. 7. Variation of the strength of phase locking with $\mathrm{CF}$, stimulus frequency and SR. A Average strength of phase locking, expressed as VS, for responses to tones presented at $70 \mathrm{~dB}$ SPL. Abscissa: stimulus frequency. Open symbols: all SRs. Open circles: $\mathrm{CFs}>4 \mathrm{kHz}$. Open squares: $\mathrm{CFs}<3 \mathrm{kHz}$. Dots indicate highly significant differences between VSs for ANFs with $\mathrm{CFs}<3 \mathrm{kHz}$ and $>4 \mathrm{kHz}$. Filled symbols: low-SR ( $\leq 1$ spike/s) ANFs. Filled squares: low-SR ANFs with $\mathrm{CFs}_{\mathrm{s}}<$ $3 \mathrm{kHz}$. Filled circles: low-SR ANFs with $\mathrm{CFs}>4 \mathrm{kHz}$. Inset: maximal VSs for ANFs in chinchilla (open symbols), cat (solid line), and guinea pig (dashed line). The maximal VSs for chinchilla at frequencies lower and higher than $800 \mathrm{~Hz}$ correspond, respectively, to the averages for $\mathrm{CFs}>4$ and $<3 \mathrm{kHz}$. Cat and guinea pig data are averages computed within 0.1-decade bins (Fig. 3 of Weiss and Rose 1988) from the original data of Johnson (1980) and Palmer and Russell (1986), respectively. B VS for responses to $2.5-\mathrm{kHz}$ tones presented at $70 \mathrm{~dB}$ SPL plotted against CF (open symbols). Filled symbols: VS averages, computed in nonoverlapping $1 / 3$-octave CF bands. Black lines: linear fits to the VS values for $\mathrm{CFs}_{3}<3,3-4$, and $>4 \mathrm{kHz}$. Gray area encompasses responses with presumably saturated VS. C Circles: rate thresholds for $2.5-\mathrm{kHz}$ tones; squares: rate thresholds $+10 \mathrm{~dB}$. Horizontal line marks $70 \mathrm{~dB} \mathrm{SPL}$, the level of the $2.5-\mathrm{kHz}$ tone stimulus. anchored unambiguously at the lowest frequencies. At those frequencies, responses are roughly synchronous with peak condensation for $\mathrm{CFs} \leq 1.5 \mathrm{kHz}$ and peak rarefaction for $\mathrm{CFs} \geq 9.5 \mathrm{kHz}$. For $\mathrm{CFs}$ between 1.9 and $7.6 \mathrm{kHz}$, responses for the lowest stimulus frequencies may coincide with rarefaction or condensation or may have intermediate phases, as previously shown (Ruggero and Rich 1987).

For ANFs with center $\mathrm{CFs} \leq 1.5$ and $\geq 9.5 \mathrm{kHz}$, all phase-frequency curves within each $\mathrm{CF}$ band were tightly clustered. In contrast, curves for CFs centered between 1.9 and $7.6 \mathrm{kHz}$ had low-frequency segments clustered around two separate loci, about 0.5 periods apart (e.g., for CFs of 1.9 and $2.4 \mathrm{kHz}$ ) and/or trajectories with low-frequency irregularities which varied substantially (e.g., for center CFs of 3 and $3.8 \mathrm{kHz}$ ). Each of the two panels of Figure 9 shows phase-frequency curves of ANFs recorded in the same ear, purposely plotted so that they coincide at the lowest frequencies. The three phase-frequency curves in Figure 9A represent ANFs with CFs in the range 2.1$2.4 \mathrm{kHz}$, which spans $0.34 \mathrm{~mm}$ (Müller et al. 2008). The curve with $\mathrm{CF}=2.15 \mathrm{kHz}$ has a constant slope, typical of ANFs with CFs in the $0.5-1.5-\mathrm{kHz}$ range (Fig. 8), whereas the curves with CFs of 2.14 and 2.4 have trajectories typical of higher CFs, with steep slopes around CF and shallow slopes for lower frequencies. Figure 9B shows curves that differ by almost exactly one period over most their extent, including CF. This anomalous behavior, illustrated in Figure 9B, has been previously described in gerbil ANFs (Ronken 1986). Therefore, to construct Figure 8, phase-frequency curves were occasionally displaced vertically by exactly one period to make them coincide with the majority population at $\mathrm{CF}$ or, in the case of $\mathrm{CFs}>3 \mathrm{kHz}$, around $2 \mathrm{kHz}$.

Average phase-frequency curves and their group delays

Figure 10 summarizes the trends of Figure 8 by presenting phase-frequency curves averaged in 21 nonoverlapping 1/3-octave CF bands (Fig. 10A) and the standard deviations of phases computed at $\mathrm{CF}$ or $2.5 \mathrm{kHz}$ (Fig. 10B). Solid and dashed lines in Figure $10 \mathrm{~A}$, respectively, indicate that the phases are tightly distributed ( $\mathrm{SD} \leq 0.25$ period) or widely scattered ( $\mathrm{SD} \geq 0.375$ period). Three trends are evident. Firstly, the overall slopes of the curves for frequencies $\leq 3 \mathrm{kHz}$ decrease systematically as a function of increasing CF. Secondly, the cumulative phase lags at $\mathrm{CF}$ (filled circles) relative to the responses at the lowest frequencies increase from about 0.75 periods for the CF band centered at $188 \mathrm{~Hz}$ to 1.5 periods for CFs around $0.8 \mathrm{kHz}$ and then remain constant in the CF range $0.8-3 \mathrm{kHz}$. Thirdly, the curvature of the phase-frequency curves changes as a function of 


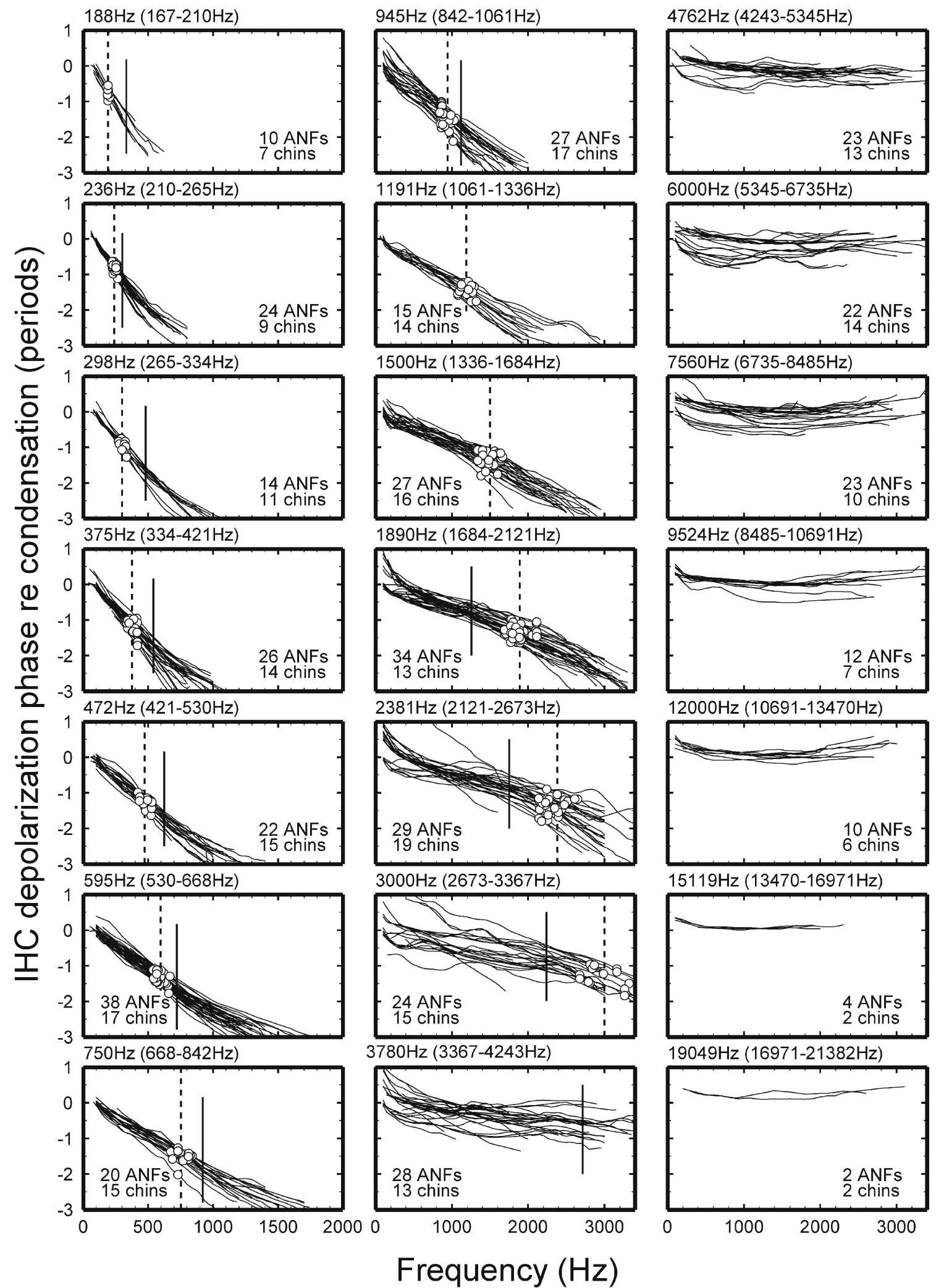

FIG. 8. Putative phase-frequency functions for IHC depolarization. Each panel presents phase-vs.-frequency functions for responses to tones, presented at $70 \mathrm{~dB} \mathrm{SPL}$, of IHCs with CFs within a 1/3-octave band (indicated at top). IHC depolarization phases were deduced from ANF excitatory phases by correction for 1-ms synaptic/neural delay. Vertical dash lines correspond to the central frequency of the band. Vertical solid lines indicate the approximate frequencies of inflection in the phase-frequency curves (open symbols in Figs. 10 and 12). Number of ANFs and chinchillas are indicated in each panel. 


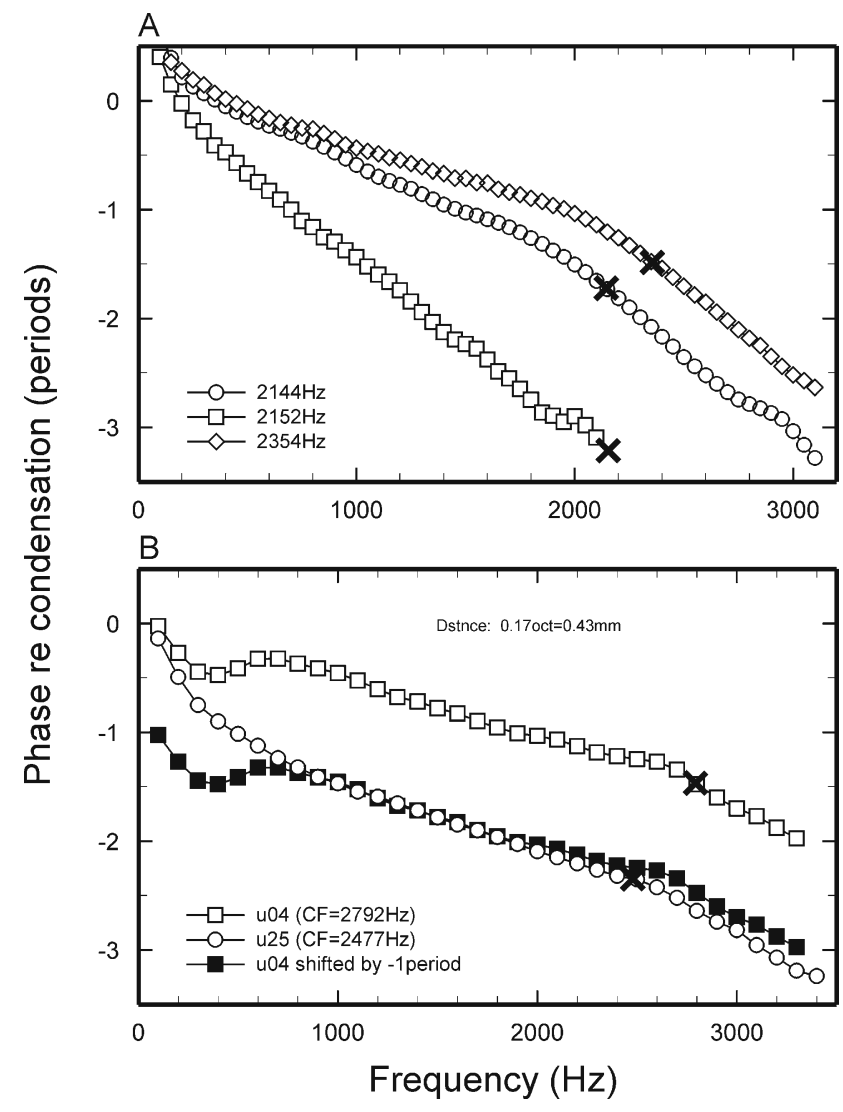

FIG. 9. Variability of phase-frequency functions in the CF transition region. ANFs with similar CFs (see legend) can have very different phase-frequency curves. Each panel presents phases of ANFs responses to tones presented at $70 \mathrm{~dB} S \mathrm{SL}$ recorded from the same auditory nerve. Multiplication symbols indicate phases at CF. A SRs 5.0, 3.7, and 92.4/s for the ANFs with CFs of 2,144, 2,152, and $2,354 \mathrm{~Hz}$, respectively. B SRs 71.5 and 109.3/s for the ANFs with CFs of 2,792 and $2,477 \mathrm{~Hz}$, respectively.

increasing $\mathrm{CF}$, from concave-upward for $\mathrm{CFs}<1 \mathrm{kHz}$ to concave-downward for $\mathrm{CFs} \geq 3 \mathrm{kHz}$.

The curvature of phase-frequency curves is more readily appreciated after detrending them (Fig. 11). Detrending consisted of rotating each of the phasefrequency curves of Figure 10A counterclockwise in proportion to the group delay measured around CF. This procedure causes phases at $\mathrm{CF}$ to equal 0 . For CFs $\leq 945 \mathrm{~Hz}$ (blue traces), detrended phases become increasingly positive as frequencies increase above CF. This occurs because the original phasefrequency curves (Fig. 10A) have lower slopes at frequencies $>\mathrm{CF}$ than at frequencies $\leq \mathrm{CF}$. For $\mathrm{CFs}$ $1.2-2.4 \mathrm{kHz}$ (black traces), the slopes of the detrended phase above $\mathrm{CF}$ become increasingly shallower. For a $\mathrm{CF}$ of $3 \mathrm{kHz}$ (red trace), phases reach a maximum near $\mathrm{CF}$ and then decrease, becoming increasingly negative. This occurs because the original phase-frequency curves (Fig. 10A) have their steepest slopes at frequencies somewhat higher than $\mathrm{CF}$.
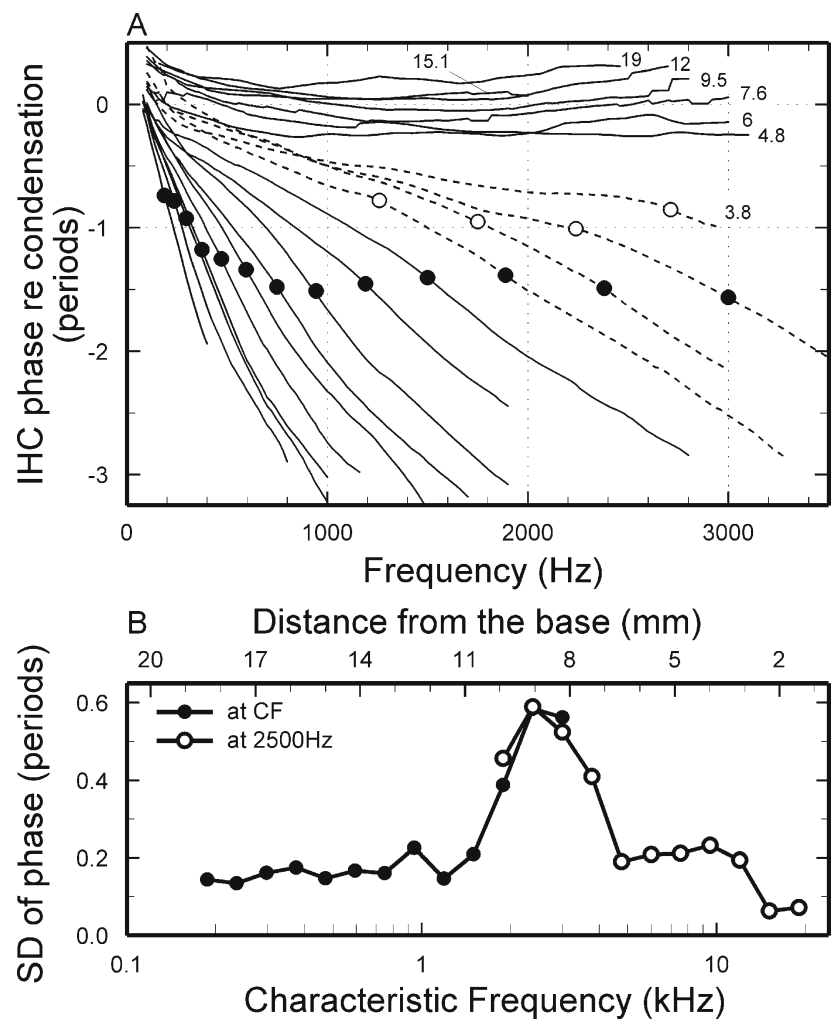

FIG. 10. Averaged IHC phase-frequency functions. A The curves are averages of the data of Figure 8 computed in 21 nonoverlapping 1/3-octave CF bands. Filled circles: phases at CF for the 13 bands with central frequency $\leq 3 \mathrm{kHz}$. Open circles: phases at the inflections of phase-frequency curves with central CFs of 1.9, 2.4, 3 , and $3.8 \mathrm{kHz}$. Dashed lines indicate data with large SDs $(>0.375)$. Numbers indicate central CFs in $\mathrm{kHz}$. B SDs of the mean phases plotted against CF. Phases measured at CF for CFs $\leq 3 \mathrm{kHz}$ (closed circles) or at $2.5 \mathrm{kHz}$ for $\mathrm{CFs} \geq 1.9 \mathrm{kHz}$ (open circles).

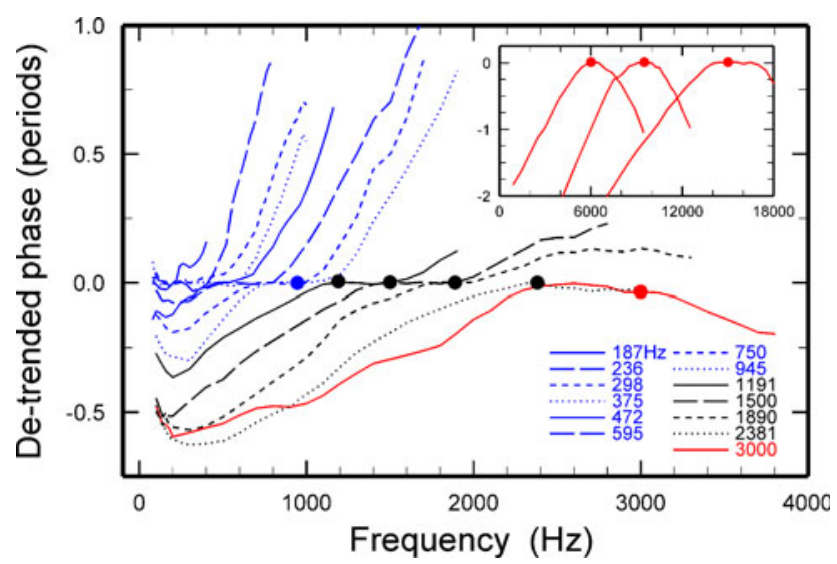

FIG. 11. Detrended phase-frequency curves of ANF and BM responses. The ANF phase-frequency curves of Figure $10 \mathrm{~A}$ were detrended by counterclockwise rotations proportional to the group delay around CF. Filled circles: phases at CFs of 0.95, 1.2, 1.5, 1.9, 2.4 , and $3 \mathrm{kHz}$. Inset: detrended phase-frequency curves for BM responses at basal sites of the chinchilla cochlea. Filled circles indicate phase at CF. BM data from Figure 1C of Ruggero et al. (2000), Figure 5 of Rhode and Recio (2000), and Figure 2 of Narayan and Ruggero (2000). 
For comparison with the detrended curves of ANFs, the inset of Figure 11 presents detrended phasefrequency curves for BM vibrations at basal sites of the chinchilla cochlea with CFs of $6,9.5$, and $15 \mathrm{kHz}$. In all three cases, the detrended BM curves resemble the curve for the $\mathrm{ANF}$ with $\mathrm{CF}=3 \mathrm{kHz}$ (red trace): They have maxima at frequencies somewhat lower than $\mathrm{CF}$ and then decrease, indicating that the steepest slopes occur at frequencies $>\mathrm{CF}$. This resemblance suggests that, for CFs 3-6 kHz, ANF and BM phase-frequency curves should be similar to those of sites with $\mathrm{CFs} \geq$ $6 \mathrm{kHz}$.

Figure 12 shows the group delays (instantaneous slopes) of the average phase-frequency curves of Figure 10A, plotted against frequency. In general, group delays reach maximum values near CF (filled circles), which decrease systematically with increasing $\mathrm{CF}$. For $\mathrm{CFs}<1 \mathrm{kHz}$, group delays are relatively constant for frequencies lower than $\mathrm{CF}$ and decrease sharply at frequencies somewhat higher than CF (open circles), corresponding to the inflections in the phase-frequency curves (solid vertical lines in Fig. 8).

\section{Inflections in phase-frequency functions and FTCs}

Figure 13 allows for comparison of the shapes of FTCs and phase-frequency curves of individual ANFs with CFs $<0.9 \mathrm{kHz}$ (upper panels), $\approx 1 \mathrm{kHz}$ (middle panels), and $>1 \mathrm{kHz}$ (lower panels). For CFs $>2 \mathrm{kHz}$, the inflections in the phase-frequency curves (arrows) occur at frequencies close to those at which FTC tails meet the tips. For ANFs with $\mathrm{CF}<0.9 \mathrm{kHz}$, the inflections in the phase-frequency functions occur at frequencies higher than CF but do not coincide

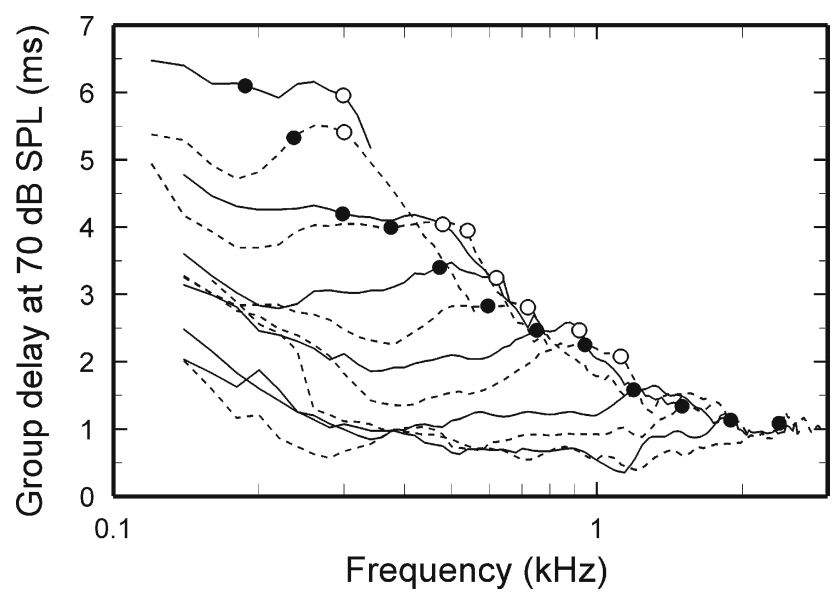

FIG. 12. Group delays of ANF responses to tones plotted against stimulus frequency. Group delays computed from the averaged phase-frequency data of Figure 10A. Filled symbols: group delays at CFs. Open symbols: group delays corresponding to inflections in phase-frequency curves. Inflection frequencies are indicated in Figures 8 and 14B. with any obvious features of the FTCs. The inflection frequencies in FTCs and phase-frequency curves, measured for 36 ANFs in which both features could be clearly identified, are plotted against each other in Figure 14A (open circles). Figure 14A shows that the inflections in FTCs and phase-frequency curves of chinchilla ANFs with $\mathrm{CFs} \geq 2 \mathrm{kHz}$ covary, with phase inflections occurring at frequencies tightly clustered around a locus (solid line) about 0.3 octave higher than the locus of FTC inflections (dashed line). Figure 14A includes comparable data for vibrations at several high-CF BM sites in chinchilla cochleae (solid triangles). The inflections in the BM data also covary, with phase inflections clustered, as for the ANF data, at frequencies about 0.3 octave higher than the FTC inflections. In addition, Figure 14A includes data for two low-CF cat ANFs (filled squares), in which the inflections of phase-frequency curves and FTCs occur at the same frequencies, higher than CF (Kiang 1984).

When the frequencies of ANF phase inflections are plotted against CF (Fig. 14B), the data points for $\mathrm{CFs} \geq 2 \mathrm{kHz}$ (open circles) tend to cluster around frequencies corresponding to -0.5 octave re $\mathrm{CF}$ (dotted line). The same line describes fairly well the trend for BM phase inflections at high-CF sites of the chinchilla cochlea (filled triangles). Figure 14B also shows the inflection frequencies for chinchilla (open diamonds) and cat (filled squares) ANFs with $\mathrm{CFs}<$ $1 \mathrm{kHz}$. In both cases, inflections occur at frequencies about 0.5 octaves above CFs (dotted line). Whereas the chinchilla ANF data of Figure 14A (open circles) are tightly clustered, indicating a high correlation between phase and FTC inflections, the same phase data plotted against CF (circles in Fig. 14B) exhibit wide scatter in the region with CFs of $2-4 \mathrm{kHz}$. This is consistent with the previously noted high variability of phase-frequency curves in that CF region (Figs. 9 and 10B).

\section{CF-specific level dependence of response phases}

The panels of Figure 15 show families of phasefrequency curves, with stimulus level as the parameter, for representative ANFs with CFs 157-2,477 Hz. Each curve indicates phases of responses to tones presented at the indicated level (30-80 $\mathrm{dB}$ SPL) or at rate threshold (dashed line), normalized to responses to tones presented at $70 \mathrm{~dB}$ SPL (horizontal dotted line). Phases vary systematically with level for frequencies lower and higher than CF (vertical dash line) but little near CF. Figure 16 illustrates population trends for the normalized phase-frequency curves (solid lines in panels $\mathrm{A}$ and $\mathrm{B}$ ): Increments in stimulus level are accompanied by phase lags for frequencies $<\mathrm{CF}$ and leads for frequencies $>$ CF. Figure 16B also shows 

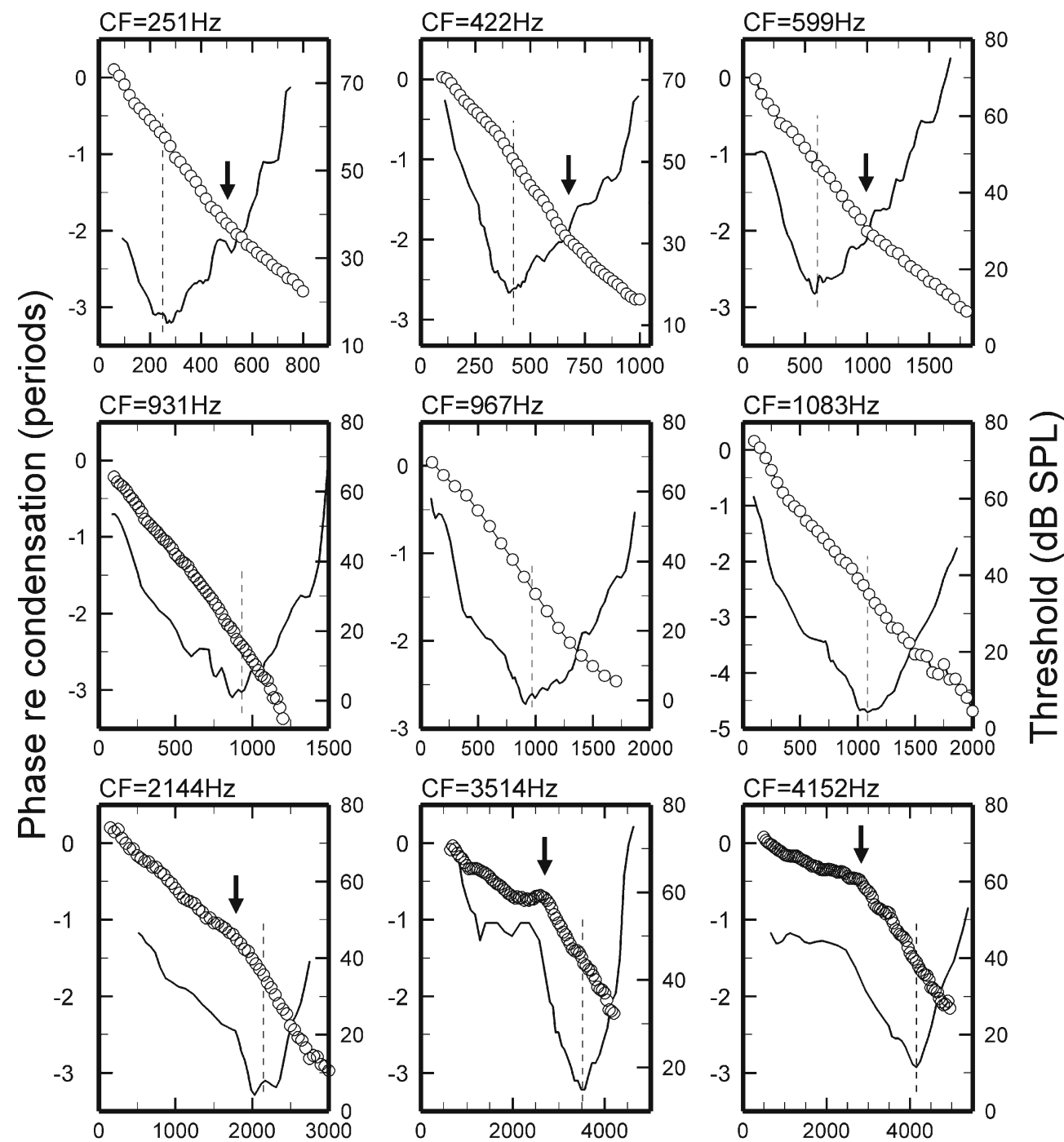

\section{Frequency $(\mathrm{Hz})$}

FIG. 13. Phase-frequency curves and FTCs for ANFs representative of those with $\mathrm{CFs}<1 \mathrm{kHz}$ (upper), $\sim 1 \mathrm{kHz}$ (center), and $>1 \mathrm{kHz}$ (bottom). Each panel shows a phase-frequency function for responses to tones presented at $70 \mathrm{~dB}$ SPL (symbols and left scale)

normalized phase-frequency curves for BM vibrations at sites of the chinchilla cochlea with CF near $10 \mathrm{kHz}$ (filled symbols), which follow a similar level-dependent pattern. That pattern was found in a vast majority of ANFs, as may be ascertained in Figure 16D, which plots the maxima and minima of a large sample of normalized phase-frequency curves such as those of panels A and B. The maxima and minima of the normalized phase-frequency curves mostly occurred at frequencies $<\mathrm{CF}$ and $>\mathrm{CF}$, respectively. The mean differences (periods $\pm \mathrm{SD}$ ) between phase maxima and minima (not shown) were similar for ANFs with $\mathrm{CFs}<$ $0.9 \mathrm{kHz}(0.22 \pm 0.16 ; n=59)$ and $0.9-2.5 \mathrm{kHz}(0.30 \pm$ $0.22 ; n=31)$ but much larger for the high-CF BM data ( 0.57 periods). and an FTC (solid line and right scale) measured in a single ANF. ANF CFs $(0.25-4.2 \mathrm{kHz})$ are indicated by vertical dash lines. Arrows mark inflections in phase-frequency curves. Abscissae indicate frequency in linear scale.

The level-dependent changes of phase-frequency functions result in corresponding level-dependent changes in near-CF group delays (Fig. 16C). The differences between group delays for responses to near-threshold and 70-dB tones expressed in CF periods (open symbols) increased systematically with increasing CF. A linear-log fit to the ANF leveldependent group-delay changes (solid line) also matches well (dashed line) the group-delay changes between $\mathrm{BM}$ responses to tones presented at 0 or 10 and $70 \mathrm{~dB}$ SPL (filled symbols, same as those of Fig. 16B) recorded at sites with CFs near $10 \mathrm{kHz}$. The level-dependent phase changes spanned frequency ranges which also varied with CF. For CFs $0.9-2.5 \mathrm{kHz}$, level-dependent changes extended down to frequen- 

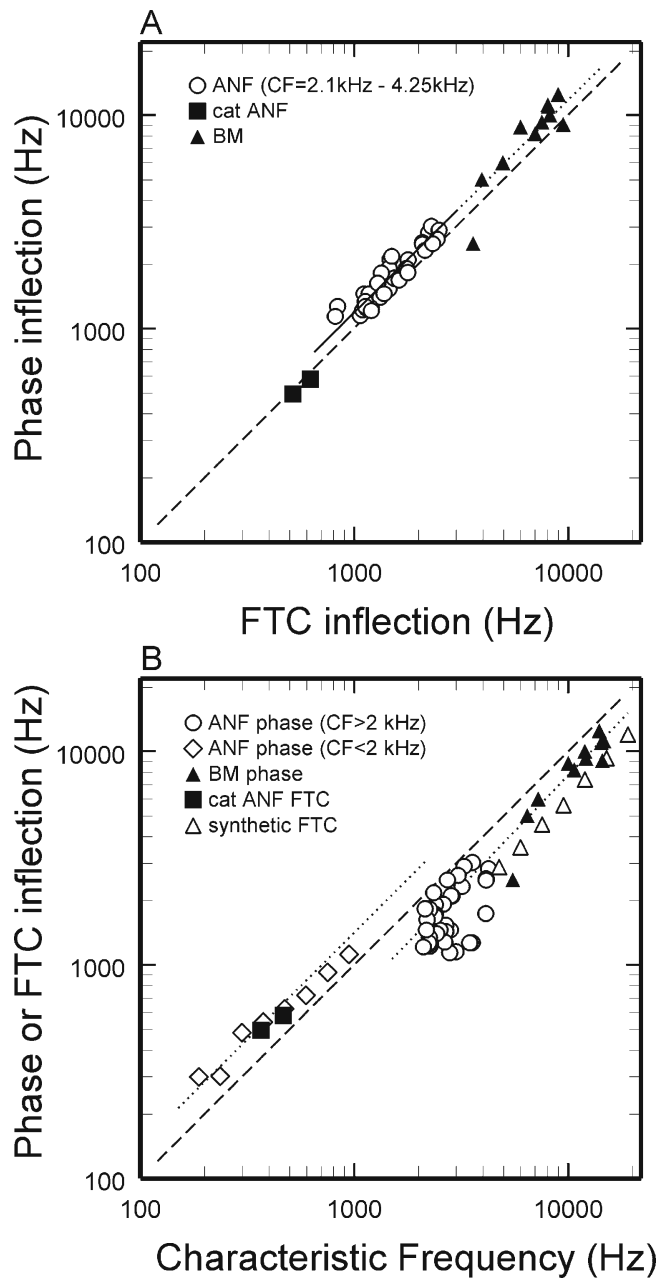

FIG. 14. Inflection frequencies of phase-frequency functions and FTCs of ANFs and BM responses in chinchilla. Open circles: data for 36 chinchilla ANFs. Filled squares: data for two cat ANFs (Fig. 12 of Kiang 1984). Filled triangles: BM data (Ruggero et al. 2000). A Inflections in phase-frequency curves plotted against inflections of ANF FTCs or BM tuning curves. Solid line is a linear regression line. Dashed line indicates equality between inflection frequencies and CF. B Frequencies of inflections in ANF or BM phase-frequency curves and ANF FTCs, plotted against CF. Open triangles: inflections in synthetic FTCs of chinchilla ANFs (from Fig. 7 of Temchin et al. 2008b). Dotted lines indicate frequencies 0.5-octave higher and lower than CF.

cies 2.5-4 octaves lower than CF but only 2 octaves for CFs $<0.9 \mathrm{kHz}$. The normalized phase-frequency curves measured at basal BM sites (filled symbols in Fig. 16B) extend over a narrow frequency range $(<1$ octave lower than $\mathrm{CF}$ ) which coincides (not shown) with the (similarly narrow) range of compressive nonlinearity (Ruggero et al. 1997; Ruggero et al. 2000). The coincidence of the ranges of level-dependent phases and CF-specific magnitude nonlinearity also applies to ANF responses, as may be ascertained by comparing Figure 2 with Figures 15 and 16A, B. However, in contrast with the BM phase and magnitude nonlinearities at basal cochlear sites, which span only a small fraction of the response bandwidth (near $\mathrm{CF}$ ), the phase and magnitude nonlinearities of ANFs with $\mathrm{CFs}<2.5 \mathrm{kHz}$ span their entire response bandwidths.

\section{DISCUSSION}

Summary of experimental findings

1. With increases in stimulus level, rate-frequency functions of ANFs with CFs lower and higher than $1 \mathrm{kHz}$, respectively, shift toward frequencies higher and lower than CF (Figs. 2 and 3).

2. The directions of the level-dependent shifts in rate-frequency functions are predicted by the asymmetries of FTCs (Fig. 4).

3. Rate-level curves for CF tones have steeper slopes than for tones with either higher or lower frequencies; for frequencies higher than CF, rate-level curves often saturate at lower rates than at CF (Fig. 5).

4. VS in chinchilla ANFs varies with frequency roughly as in cat and guinea pig (inset of Fig. 7A). In chinchilla, the bandwidth of VS for $\mathrm{CFs}<3 \mathrm{kHz}$ exceeds, by 0.75 octaves, the bandwidth for $\mathrm{CFs}>4 \mathrm{kHz}$. For $\mathrm{CFs}<3 \mathrm{kHz}$ (but not for $\mathrm{CFs}>$ $4 \mathrm{kHz}$ ), the bandwidth of VS is larger for ANFs with low SRs $(\leq 1 \mathrm{spikes/s)}$ than for high SRs (Fig. 7A).

5. For $\mathrm{CFs}<1 \mathrm{kHz}$, phase-frequency curves have a steep low-frequency segment flanking $\mathrm{CF}$ and a shallower segment at higher frequencies. For CFs $\geq$ $3 \mathrm{kHz}$, they have a shallow low-frequency segment and a steep segment around CF (Figs. 3, 8, 10, and $11)$.

6. The lower bound of the CF region (shaded area in Fig. 3) where phase-frequency curves change shape, $1 \mathrm{kHz}$, coincides with the $\mathrm{CF}$ at which the level-dependent frequency shifts of rate-frequency curves change direction, FTCs flip their asymmetries (Temchin et al. 2008b), and the frequency glides of ANF impulse responses change their polarity (Temchin et al. 2005).

7. The cumulative phases at CF of IHC depolarization (deduced from ANF phases) increase from about 0.75 period re condensation for $\mathrm{CFs}$ around $188 \mathrm{~Hz}$ to 1.5 periods for CFs around $750 \mathrm{~Hz}$ and then remain constant for CFs up to $3 \mathrm{kHz}$ (Figs. 8 and 10).

8. ANF phase-frequency curves exhibit greater variability and more irregularities in the region with CFs $1.9-4.8 \mathrm{kHz}$ than elsewhere (Figs. 8 and 10).

9. As stimulus level is raised, the phases of responses to tones with frequency $<\mathrm{CF}$ and $>\mathrm{CF}$, respectively, increasingly lag and lead lower-level responses (Figs. 15 and 16). 


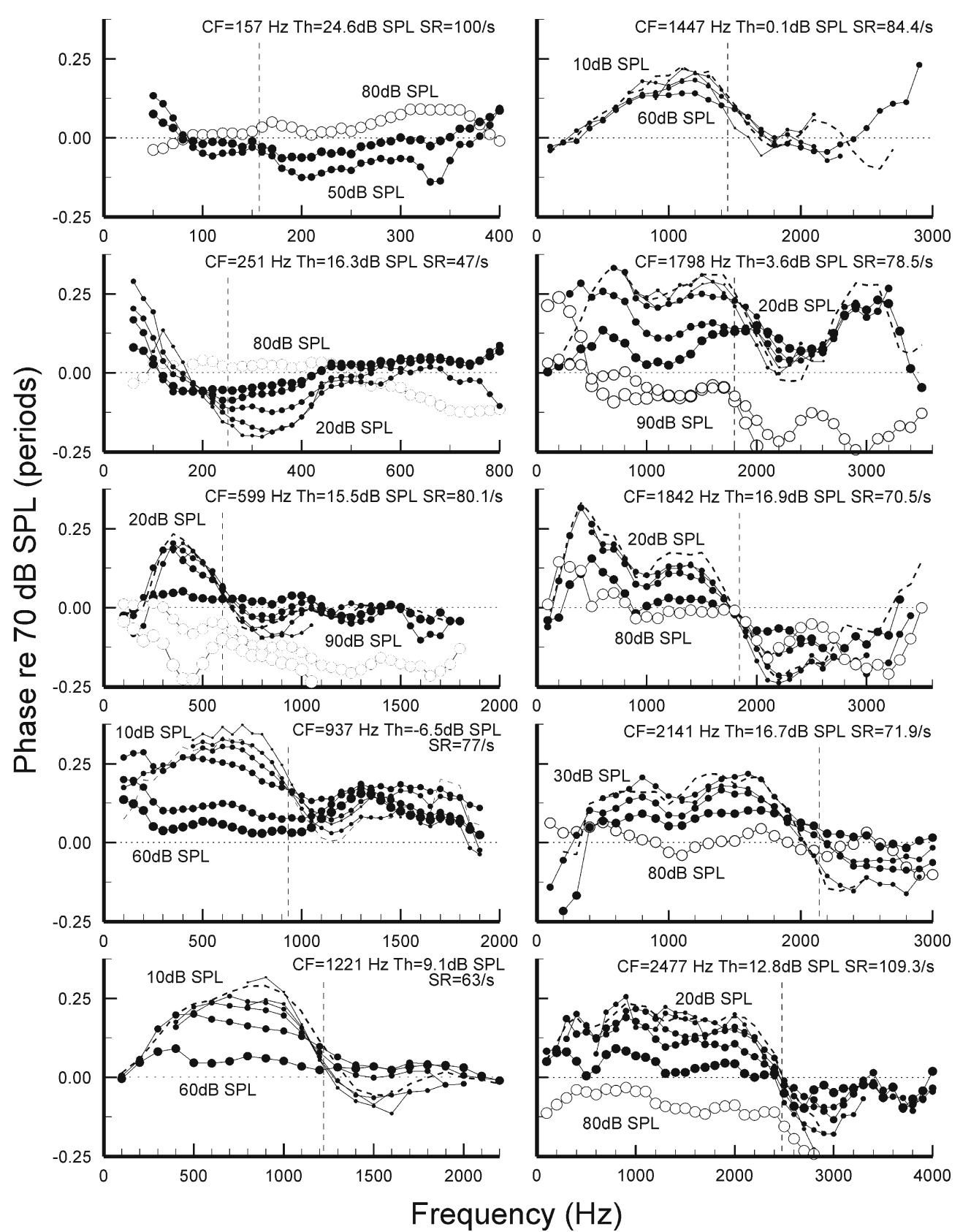

FIG. 15. Level dependence of phase-frequency curves in several ANFs. Each panel represents responses of a single ANF with CF, CF threshold, and SR indicated at top right. Horizontal dotted lines indicate the reference phases (for responses to 70-dB SPL tones).

10. The CF-specific phase and magnitude nonlinearities of ANFs with $\mathrm{CFs}<2.5 \mathrm{kHz}$ span their entire response bandwidths (Figs. 4, 5, and 15).

11. Group delays are maximal near CF (Fig. 12) and, near $\mathrm{CF}$, decrease as a function of increasing stimulus level (Fig. 16C).

12. For $\mathrm{CFs}<1 \mathrm{kHz}$, the frequencies of inflection in phase-frequency curves occur at frequencies approximately 0.5 octave higher than CF (Fig. 14B). For CFs $\geq 2-3 \mathrm{kHz}$, the frequencies of
Positive phases indicate leads relative to the phase at $70 \mathrm{~dB}$ SPL. Dashed lines: responses at rate threshold. The sizes of the filled symbols vary monotonically with stimulus level (10-70 dB SPL).

Open symbols mark levels higher than $70 \mathrm{~dB}$.

inflection occur at frequencies approximately 0.5 octave lower than CF (Fig. 14B) and are highly correlated with the frequencies of inflection in the lower limbs of FTCs (Figs. 13 and 14A).

13. The variability of response phases (Figs. 10B and 17 ), the gradients of phase with respect to $\mathrm{CF}$ or cochlear place (Figs. 10A and 17), and VS (Figs. 7B and 17) undergo rapid transitions in the $1.9-4.8-\mathrm{kHz}$ CF region, where three other 


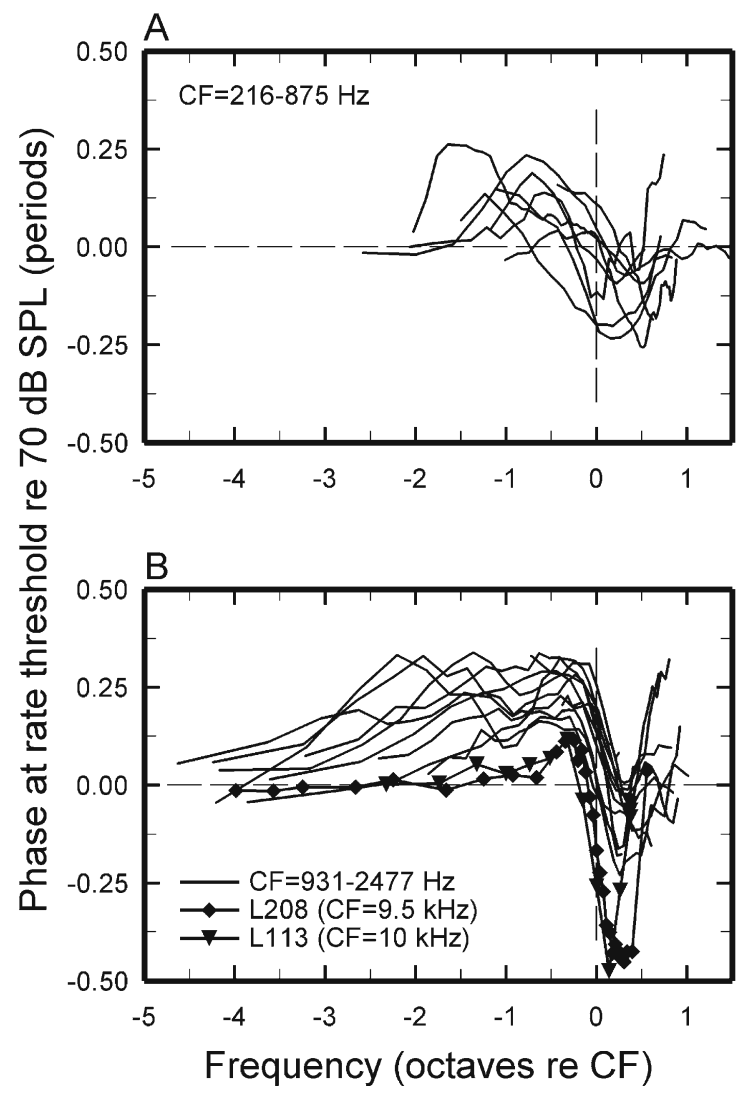

FIG. 16. Level-dependent changes of $A N F$ and $B M$ response phases. A, B Phase-frequency curves for threshold responses of ANFs normalized to phases of responses to $70-\mathrm{dB}$ tones. Tone frequency is expressed in octaves relative to CF (vertical dashed line). Positive values indicate phase leads relative to responses to $70-\mathrm{dB}$ tones. For comparison with ANF data, B shows level-dependent changes for BM responses at basal sites of the chinchilla cochlea (filled symbols) measured between 0 or 10 dB SPL (L208 and L113,
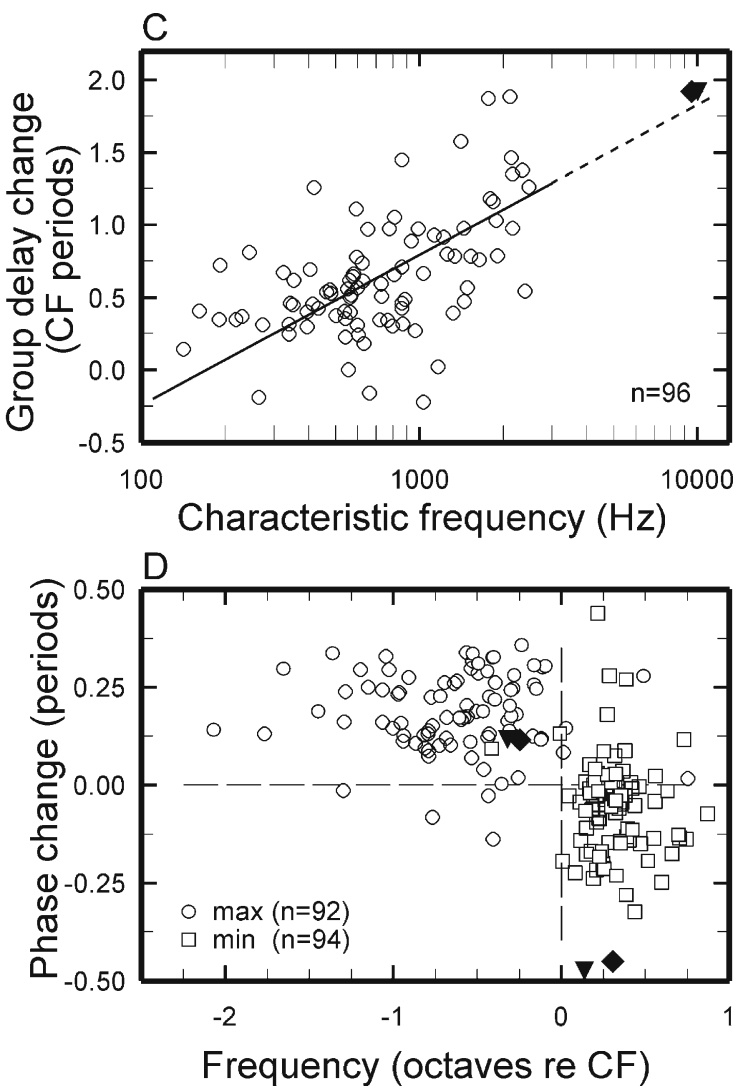

respectively) and $70 \mathrm{~dB}$ SPL. CFs 216-875 Hz (A); CFs 9312,477 Hz (B). C Near-CF group delay changes with level of ANF (open symbols) and BM responses at basal sites (filled symbols) plotted against CF. D Maxima and minima of normalized phasefrequency curves (circles and squares, respectively) plotted against stimulus frequency (expressed relative to CF). Open symbols: ANF data. Filled symbols: BM data. BM data from Ruggero et al. (1997) and Ruggero et al. (2000).

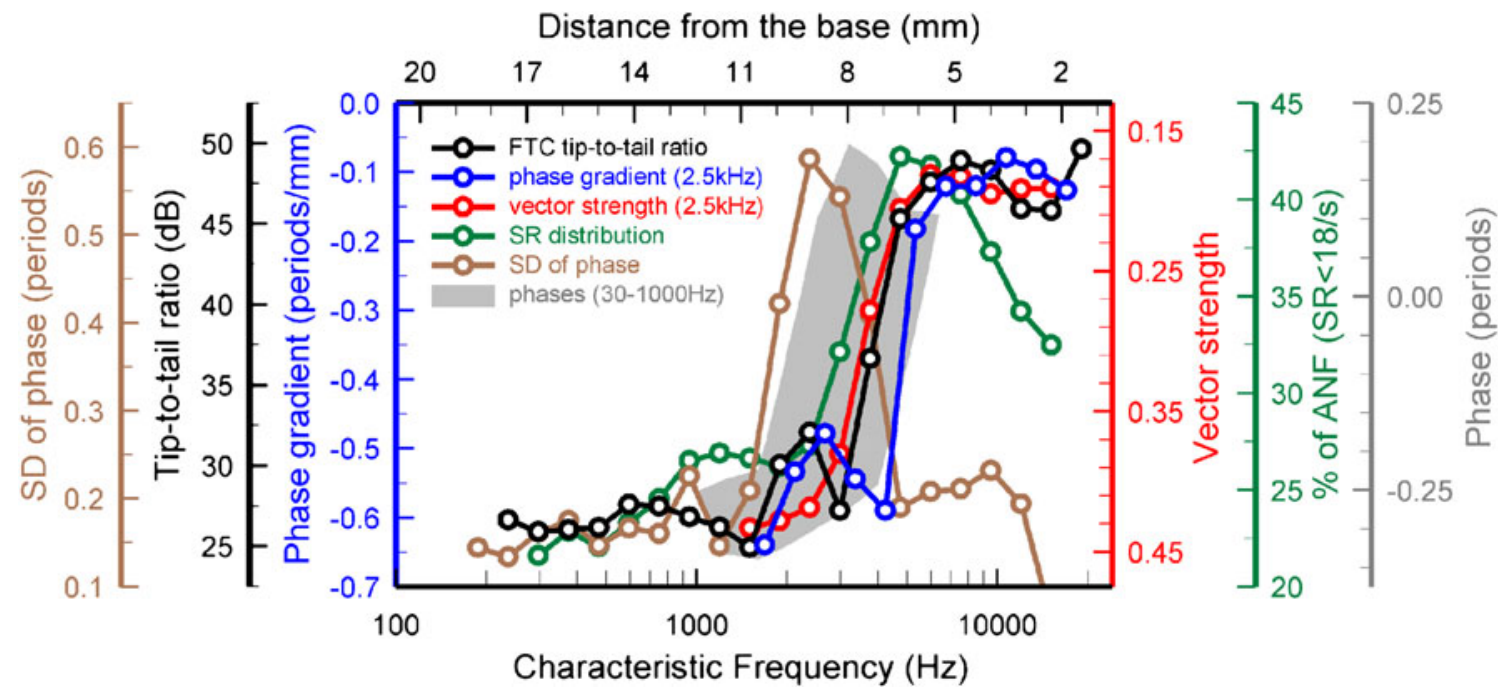

FIG. 17. Coincidence at the $2-5-\mathrm{kHz}$ CF region of transitions in several properties of ANFs. Brown: SD of phases (Fig. 10B). Black: tip-to-tail ratio of FTCs measured at 1.5 octave re CF (Fig. 2 of Temchin et al. 2008b). Blue: phase spatial gradient for $2.5-\mathrm{kHz}$ tones (Fig. 10A), i.e., phase difference between adjacent CF bands, expressed re corresponding cochlear distance. Red: average vector strengths of responses to 2,500-Hz tones (Fig. 6B). Green: percent of ANFs with $\mathrm{SRs}<18 / \mathrm{s}$ (Fig. 1C of Temchin et al. 2008a). Gray shading: IHC phases re BM of $30-1,000 \mathrm{~Hz}$ tones (Fig. 13 of Ruggero and Rich 1987). 
properties of ANFs also undergo pronounced transitions (Fig. 17): the distribution of SRs (Temchin et al. 2008a), the slopes of the lower limbs of FTCs (Temchin et al. 2008b), and the phases of responses to low-frequency tones (Ruggero and Rich 1987).

\section{Level dependence of ANF response rates}

The level-dependent frequency shifts of rate-frequency curves of chinchilla ANFs (Fig. 2), including their transition at a $\mathrm{CF}$ of about $1 \mathrm{kHz}$ (Fig. 3), are comparable to those in squirrel monkey (Anderson et al. 1971; Rose et al. 1971) and also clearly related to level-dependent frequency shifts in responses to noise in cat (Evans 1977; Louage et al. 2004). For frequencies $>$ CF, ANF rate-level functions have lower slopes and/or saturation rates than for $\mathrm{CF}$ stimuli in chinchilla (Fig. 5; Jackson and Relkin 1998), cat (Sachs and Abbas 1974), and guinea pig (Cooper and Yates 1994). For frequencies < CF, slopes and/or saturation rates in chinchilla are also lower than for CF (Fig. 5), with precedents in two low-CF ANFs (Figs. 2B of Jackson and Relkin 1998 and Fig. 4 of Cooper and Rhode 1997). This finding is consistent with the fact that slopes of putative BM input-output functions at CF (derived from ANF rate-level functions on the assumption that $\mathrm{BM}$ responses grow compressively at CF but linearly at frequencies $<\mathrm{CF}$ ) could not be computed for $\mathrm{CFs}<1.5 \mathrm{kHz}$ (Cooper and Yates 1994).

\section{ANF phase-frequency curves}

The shapes of phase-frequency curves in chinchilla ANFs and their variation with CF (Figs. 8, 10A and 11) are similar to those in cat (Allen 1983; Carney et al. 1999; Pfeiffer and Molnar 1970; van der Heijden and Joris 2003) and probably also squirrel monkey (Anderson et al. 1971; Geisler et al. 1974). The finding of a constant cumulative phase at $\mathrm{CF}$ for peak depolarization of chinchilla IHCs with CFs $0.75-3 \mathrm{kHz}$ (Figs. 7 and 10) is unprecedented for any species. Most of the previous comparable studies neither reported phases relative to stimulus pressure nor included sufficient information to address the variation of CF phase as a function of CF (Anderson et al. 1971; Geisler et al. 1974; Palmer and Shackleton 2009; Pfeiffer and Molnar 1970). Previous phase data for chinchilla ANFs with CFs $\leq 800 \mathrm{~Hz}$ (Fig. 9 of Ruggero and Rich 1987) are consistent with the IHC phasefrequency curves of Figure 10A. In contrast with results for chinchilla, the phase lags at $\mathrm{CF}$ of putative IHC depolarization in cat increase monotonically in the entire CF range $0.2-5 \mathrm{kHz}$ (Fig. 5 of van der Heijden and Joris 2006). The discrepancy may be smaller than it appears: Personal communications from M. van der Heijden and P. X. Joris indicate that delays involved in their spike-recording system amounting to $\sim 200 \mu$ s were not compensated for and must have introduced phase lags proportional to frequency.

\section{Level dependence of ANF response phases}

The level dependence of ANF responses phases around $\mathrm{CF}$ is consistent with previous results for chinchilla (Fig. 13C of Recio-Spinoso et al. 2005) as well as squirrel monkey (Anderson et al. 1971), guinea pig (Palmer and Shackleton 2009), and cat (van der Heijden and Joris 2003; Carney and Yin 1988). Similarly, the frequency range of level dependence of phase in chinchilla ANFs with $\mathrm{CFs}<2.5 \mathrm{kHz}$, spanning 2-4 octaves, is similar to the corresponding ranges for squirrel monkey ANFs (Anderson et al. 1971). However, the pattern of level dependence of response phases in chinchilla ANFs with $\mathrm{CFs}>1 \mathrm{kHz}$ (Figs. 15 and 16) is the reverse of the pattern found by Rhode and Cooper (1997) in ANFs of the same species. The discrepancy may reflect differences in stimulus levels (typically $\leq 70 \mathrm{~dB}$ SPL here vs. $>70 \mathrm{~dB}$ SPL for Rhode and Cooper 1997).

\section{Transition of ANF response properties at the $1-\mathrm{kHz}$ CF region}

Both the magnitudes and phases of chinchilla ANF responses to tones undergo pronounced transitions in the cochlear region with $\mathrm{CF}$ near $1 \mathrm{kHz}$ (Figs. 3 and 11). The lower bound of the CF region where phasefrequency curves undergo shape changes (gray shading in Fig. 3) coincides with the CF, $1 \mathrm{kHz}$, around which level-dependent shifts of rate-frequency curves change direction, FTCs flip their asymmetries (Temchin et al. 2008b), and the frequency glides of ANF impulse responses switch polarity (Temchin et al. 2005). The FTC asymmetries directly predict the direction, but not the fine detail, of the level-dependent frequency shifts in rate-frequency functions (Fig. 4). The FTCs of cat ANFs with $\mathrm{CFs} \leq 1 \mathrm{kHz}$ may have upper limb inflections at frequencies about 0.5 octave higher than $\mathrm{CF}$, which coincide with inflections in phase-frequency curves (filled black squares in Fig. 14; see Fig. 12 of Kiang 1984). Comparable inflections are not obvious in FTCs of chinchilla ANFs with $\mathrm{CF} \leq 1 \mathrm{kHz}$ (Temchin et al. 2008b), but their phase-frequency curves do exhibit inflections at frequencies about 0.5 octaves higher than CF (Fig. 14B). The correlated features of ANF FTCs, phase-frequency curves (Figs. 13 and 14), and fre- 
quency glides of impulse responses (Fig. 3) probably reflect BM counterparts (Temchin et al. 2009).

Transition of ANF response properties at the $2-5-\mathrm{kHz}$ CF region

A striking collection of properties of chinchilla ANFs undergo transitions in the cochlear region with CFs $2-5 \mathrm{kHz}$ (Fig. 17). In chinchilla, inflections in phasefrequency functions for ANFs with $\mathrm{CFs} \geq 2.1 \mathrm{kHz}$ (Figs. 13 and 14A) coincide with inflections in FTCs. This finding, apparently not previously reported in any species, is consistent with the twin facts that phase-frequency curves for basal BM sites (filled black triangles in Fig. 14) and FTCs of ANFs with $\mathrm{CFs} \geq 4.8 \mathrm{kHz}$ (open triangles in Fig. 14B) both exhibit inflections at frequencies about 0.5 octave lower than CF in chinchilla. The correlated inflections in FTCs and phase-frequency curves of individual ANFs (Fig. 13) are clearly related to the transitions in the 3-4-kHz CF region of the shapes of FTCs (black circles in Fig. 17) and of the spatial gradient of response phases (blue circles in Fig. 17). Since frequency tuning has a largely mechanical origin, the correlated inflections in phase-frequency functions and their transitions in the $3-4-\mathrm{kHz}$ CF region (which allegedly can be predicted from ANF FTCs; Temchin et al. 2009) probably also arise in BM vibrations. In contrast, the jump of VS at CFs between 3 and $4 \mathrm{kHz}$ probably reflects a transition in intrinsic properties of the IHCs and/or their synapses. This inference arises from the fact that, for sufficiently high CFs (Fig. 6, right-side panels), the DC component has a wider frequency response than the AC component (middle row). This is reasonable since the DC component more closely reflects the full bandwidth of the mechanical input to the IHC stereocilia than the AC component, which additionally reflects low-pass filtering by the IHC basolateral membrane (Palmer and Russell 1986) and by synaptic processes (Weiss and Rose 1988). Finally, the polarity flip of the phases of ANF responses to $30-1,000 \mathrm{~Hz}$ tones (gray shaded area in Fig. 17, as described by Ruggero and Rich 1983 and Ruggero and Rich 1987) and the increased variability of phase-frequency curves (Fig. 10B and brown symbols in Fig. 17), for which it is partly responsible, probably reflect micromechanical processes interposed between $\mathrm{BM}$ vibrations (see Fig. 6 of Ruggero et al. 2000) and the deflection of IHC stereocilia (Ruggero and Rich 1987).

A transition in the distribution of ANF SRs at the $3-4-\mathrm{kHz}$ CF region (green symbols in Fig. 17), accompanied by differences in the slopes and saturation rates of rate-level functions, was previously noted in gerbil (Ohlemiller et al. 1991). The findings in chinchilla that VS bandwidth is larger for ANFs with
$\mathrm{CFs}<3 \mathrm{kHz}$ than for $\mathrm{CFs}>4 \mathrm{kHz}$ and that there is an inverse correlation between VS and SR have precedents in cat (Johnson 1980; Joris et al. 1994; Joris and Yin 1992). The fact that the inverse correlation is restricted to ANFs with $\mathrm{CFs}<3 \mathrm{kHz}$ is reminiscent of results for responses to noise of cat ANFs (Louage et al. 2004). The difference between the bandwidths of VS for ANFs with $\mathrm{CFs}<3$ and $>4 \mathrm{kHz}$ may help to explain why the bandwidth of phase locking to the envelope of sinusoidally amplitude-modulated CF carriers in high-CF ANFs is lower, by 0.8 octave, than the bandwidth of phase locking to pure tones of lowCF ANFs (see Fig. 19 of Joris and Yin 1992). The variation of VS and SR as functions of $\mathrm{CF}$, as well as the inverse relation between SR and VS for $\mathrm{CFs}<$ $3 \mathrm{kHz}$ (only), all suggest tonotopic variations in IHC and/or synaptic function (Johnson et al. 2008; Meyer et al. 2009). Of special interest is that correlated morphological (shape of ribbon synapses) and functional (calcium dependence of neurotransmitter release) features of IHC synapses differ between basal and apical regions of the gerbil cochlea (Johnson et al. 2008).

Level dependence of response rates of ANFs in relation to $\mathrm{IHC}$ receptor potentials and cochlear vibrations at the apex of the cochlea

The similarity of the level dependence of response rates of low-CF ANFs in chinchilla and other species argues strongly against the possibility that the absence of level dependence of mechanical tuning at apical sites of the chinchilla cochlea (Cooper and Rhode 1997; Rhode and Cooper 1996) is a peculiarity of this species. At least at the base of the cochlea, the tuning of ANFs (Narayan et al. 1998; Ruggero et al. 2000; Temchin et al. 2008b) and IHC tuning curves (Kössl and Russell 1992; Sellick et al. 1983) reflect BM tuning. Furthermore, there is no evidence disputing the conclusion that, at the apex of the cochlea, the frequency tuning of IHCs (Dallos 1985, 1986) "results more from the micromechanical properties of the cochlea than from intrinsic properties of the cells themselves" (Kros 1996). Therefore, it is likely that the CF-specific nonlinearities illustrated in Figures 2 and 5 are present at the input to the stereocilia of apical IHCs and that their antecedents in organ of Corti vibrations in chinchilla were not detected because of abnormalities due to surgical damage (such as puncturing of Reissner's membrane; Dong and Cooper 2006). In fact, some recordings at apical cochlear sites in chinchilla (Fig. 2.3D of Cooper 2004) and guinea pig (Dong and Cooper 2001; Zinn et al. 2000) give hints of CF-specific nonlinearity. Another possibility is that a micromechanical "second filter" is interposed between the vibrations of the BM/organ of 
Corti/tectorial membrane complex and the excitation of ANFs (Dong and Cooper 2006).

At the base of the cochlea, the level dependence of BM tuning and its vulnerability to noxious agents are tightly linked to CF-specific nonlinearities. Thus, furosemide causes $\mathrm{BM}$ responses to resemble those elicited in normal cochleae by intense stimuli: The gains at $\mathrm{CF}$ are decreased, frequency tuning deteriorates, and BFs shift to lower frequencies (Ruggero and Rich 1991). Furosemide induces similar changes in the FTCs of ANFs with $\mathrm{CFs}>4 \mathrm{kHz}$ (Sewell 1984). However, in the case of ANFs with $\mathrm{CFs}<1 \mathrm{kHz}$, furosemide shifts BFs to frequencies higher than $\mathrm{CF}$ (Sewell 1984). The level-dependent frequency shifts of rate-frequency curves (Figs. 2, 3 and 4) suggest an explanation for this effect: furosemide, as well as increases of stimulus level, reduces the amplification of cochlear vibrations, raising BFs at the apex but lowering them at the base.

Similarities and differences between the phases of low-CF ANF/IHC responses and cochlear vibrations

For CFs of 500-600 Hz, the putative phases of IHC depolarization vary roughly linearly with frequency (Figs. 8 and 10) and closely match (not shown) the phases of peak velocity of tectorial membrane vibrations toward scala vestibuli (Rhode and Cooper 1996). Similarly, ANF responses in both chinchilla and cat (Allen 1983) and BM responses at basal sites of the cochlea in chinchilla (e.g., Rhode 2007), as well as other species, all exhibit maximal group delays near (or slightly higher than) CF. Also similarly, the "concave downward" shape of phase-frequency curves for $\mathrm{CFs} \geq 3 \mathrm{kHz}$ resembles the shape of $\mathrm{BM}$ phase-frequency curves at basal sites of the cochlea (e.g., Rhode 2007), and the group delays at CF of high-CF chinchilla ANFs are consistent with their counterparts in BM vibrations (Temchin et al. 2005). Thus, in all these respects, IHC and ANF responses appear to reflect directly their BM counterparts.

The level dependencies of the phases of responses to near-CF tones are qualitatively similar in low-CF chinchilla ANFs (Figs. 15 and 16) and guinea pig IHCs (Cheatham and Dallos 2001; Dallos 1986) and in $\mathrm{BM}$ vibrations at basal sites of the chinchilla cochlea (Rhode 2007; Ruggero et al. 1997, 2000). On the assumption that the frequency ranges of $\mathrm{BM}$ compressive growth and level-dependent phase changes should coincide (as is the case at basal cochlear sites), it is reasonable that compression in vibrations at apical sites of the chinchilla cochlea span several octaves around CF (Cooper and Rhode 1997; Rhode and Cooper 1996), i.e., similar to the ranges of level-dependent phase changes in low-CF chinchilla
ANFs (Figs. 16A and B) and guinea pig IHCs (Dallos 1986). However, most importantly, the level dependencies of the phases of responses around $\mathrm{CF}$ of low-CF chinchilla ANFs and of vibrations at apical sites of the chinchilla cochlea have opposite polarities (Rhode and Cooper 1996).

Conclusions regarding the mechanical bases of ANF responses to tones in apical regions of the chinchilla cochlea

1. The similarity between the properties of low-CF ANFs of chinchilla and other species argues strongly against the possibility that the apex of the chinchilla cochlea is exceptional.

2. The level-dependent frequency shifts in ratefrequency functions of low-CF chinchilla ANFs contrast with the level-independent tuning of cochlear vibrations at low-CF sites in chinchilla.

3. The pattern of level dependence of the phases of chinchilla ANF responses around CF is the reverse of the pattern of apical vibrations at apical sites of the chinchilla cochlea.

4. The level-dependent properties of response rates and phases of chinchilla ANFs with CFs $\leq 1 \mathrm{kHz}$ are CF specific, thus suggesting that they are not intrinsic in IHC receptor potentials or synaptic processes but rather reflect the mechanical input to the IHCs.

5. The ANF data suggest that, in contrast with BM vibrations at basal cochlear sites, the CF-specific phase and magnitude nonlinearities of apical vibrations span their entire response bandwidths.

\section{ACKNOWLEDGMENTS}

Many thanks to Philip Joris and Nigel Cooper for their helpful comments. We were supported by Grants from the NIH (DC-000419) and the Hugh Knowles Center.

\section{REFERENCES}

Allen JB (1983) Magnitude and phase-frequency response to single tones in the auditory nerve. J Acoust Soc Am 73:2071-2092

Anderson DJ, Rose JE, Hind JE, Brugge JF (1971) Temporal position of discharges in single auditory nerve fibers within the cycle of a sine-wave stimulus: frequency and intensity effects. J Acoust Soc Am 49:1131-1139

Buunen TJ, Rhode WS (1978) Responses of fibers in the cat's auditory nerve to the cubic difference tone. J Acoust Soc Am 64:772-781

Carney LH, Yin TC (1988) Temporal coding of resonances by lowfrequency auditory nerve fibers: single-fiber responses and a population model. J Neurophysiol 60:1653-1677 
Carney LH, Mcduffy MJ, Shekhter I (1999) Frequency glides in the impulse responses of auditory-nerve fibers. J Acoust Soc Am 105:2384-2391

Cheatham MA, Dallos P (2001) Inner hair cell response patterns: implications for low-frequency hearing. J Acoust Soc Am 110:2034-2044

Cooper NP (2004) Compression in the peripheral auditory system. In: Bacon SP, Fay RR, Popper AN (eds) Compression: from cochlea to cochlear implant. Springer, New York, pp 18-61

CoOper NP (2006) Mechanical preprocessing of amplitudemodulated sounds in the apex of the cochlea. ORL J Otorhinolaryngol Relat Spec 68:353-358

Cooper NP, RHode WS (1992) Basilar membrane mechanics in the hook region of cat and guinea-pig cochleae: sharp tuning and nonlinearity in the absence of baseline position shifts. Hear Res 63:163-190

Cooper NP, Rhode WS (1995) Nonlinear mechanics at the apex of the guinea-pig cochlea. Hear Res 82:225-243

Cooper NP, RHODE WS (1996) Fast travelling waves, slow travelling waves and their interactions in experimental studies of apical cochlear mechanics. Audit Neurosci 2:289-299

Cooper NP, Rhode WS (1997) Mechanical responses to two-tone distortion products in the apical and basal turns of the mammalian cochlea. J Neurophysiol 78:261-270

CoOper NP, YATES GK (1994) Nonlinear input-output functions derived from the responses of guinea-pig cochlear nerve fibres: variations with characteristic frequency. Hear Res 78:221-234

Dallos P (1985) Response characteristics of mammalian cochlear hair cells. J Neurosci 5:1591-1608

Dallos P (1986) Neurobiology of cochlear inner and outer hair cells: intracellular recordings. Hear Res 22:185-198

Dong W, Cooper NP (2001) Assessing the physiological condition of the cochlea during mechanical investigations of the apical cochlea. J Physiol 526P:S202

Dong W, CoOPER NP (2006) An experimental study into the acoustomechanical effects of invading the cochlea. J R Soc Interface 3:561-571

Evans EF (1977) Frequency selectivity at high signal levels of single units in cochlear nerve and nucleus. In: Evans EF, Wilson JP (eds) Psychophysics and physiology of hearing. Academic, London, pp 185-192

GeIsler CD, Rhode WS, Kennedy DT (1974) Responses to tonal stimuli of single auditory nerve fibers and their relationship to basilar membrane motion in the squirrel monkey. J Neurophysiol 37:1156-1172

GoldBerg JM, BRown PB (1969) Response of binaural neurons of dog superior olivary complex to dichotic tonal stimuli: some physiological mechanisms of sound localization. J Neurophysiol 32:613-636

HARRIS DM (1979) Action potential suppresion, tuning curves and thresholds: comparison with single fiber data. Hear Res 1:133154

JaCksOn BS, ReLKIN EM (1998) A frequency-dependent saturation evident in rate-intensity functions of the chinchilla auditory nerve. Hear Res 126:75-83

Johnson DH (1980) The relationship between spike rate and synchrony in responses of auditory-nerve fibers to single tones. J Acoust Soc Am 68:1115-1122

Johnson SL, Forge A, Knipper M, Munkner S, Marcotti W (2008) Tonotopic variation in the calcium dependence of neurotransmitter release and vesicle pool replenishment at mammalian auditory ribbon synapses. J Neurosci 28:7670-7678

Johnstone JR, Alder VA, Johnstone BM, Robertson D, Yates GK (1979) Cochlear action potential threshold and single unit thresholds. J Acoust Soc Am 65:254-257

JoRIS PX, YIN TC (1992) Responses to amplitude-modulated tones in the auditory nerve of the cat. J Acoust Soc Am 91:215-232
Joris PX, SMITH PH, YIN TC (1994) Enhancement of neural synchronization in the anteroventral cochlear nucleus. II. responses in the tuning curve tail. J Neurophysiol 71:1037-1051

KIANG NY (1984) Peripheral neural processing of auditory information. In: Darian-Smith I (ed) The nervous system. volume III: sensory processes, part 2. American Physiological Society, Bethesda, pp 639-674

Kiang NY, Moxon EC, Levine RA (1970) Auditory-nerve activity in cats with normal and abnormal cochleas. In: Wolstenholme GEW, Knight J (eds) Sensorineural hearing loss. Churchill, London, pp 241-273

KIm DO, Molnar CE (1979) A population study of cochlear nerve fibers: comparison of spatial distributions of average-rate and phase-locking measures of responses to single tones. J Neurophysiol 42:16-30

Kim DO, Siegel JH, Molnar CE (1980) Postmortem effects and species differences for acoustic input characteristics at the eardrum of the chinchilla and the cat. Soc Neurosci Abst 6:41

KössL M, Russell IJ (1992) The phase and magnitude of hair cell receptor potentials and frequency tuning in the guinea pig cochlea. J Neurosci 12:1575-1586

Kros CJ (1996) Physiology of mammalian hair cells. In: Dallos P, Popper AN, Fay RR (eds) The cochlea. Springer, New York, pp 318-385

Liberman MC, Kiang NY (1978) Acoustic trauma in cats. Cochlear pathology and auditory-nerve activity. Acta Oto-laryngol Suppl 358:1-63

Liberman MC, KIANG NY (1984) Single-neuron labeling and chronic cochlear pathology. IV. Stereocilia damage and alterations in rate- and phase-level functions. Hear Res 16:75-90

LitTLEFIELD WM (1973) Investigation of the linear range of the peripheral auditory system. D.Sci. dissertation, Washington University, St. Louis

Louage DH, Van Der Heijden M, Joris PX (2004) Temporal properties of responses to broadband noise in the auditory nerve. J Neurophysiol 91:2051-2065

MARdia KV, JuPP PE (2000) Directional statistics. Wiley, Chichester

Meyer AC, Frank T, Khimich D, Hoch G, Riedel D, Chapochnikov NM, Yarin YM, Harke B, Hell SW, Egner A, Moser T (2009) Tuning of synapse number, structure and function in the cochlea. Nat Neurosci 12:444-453

MÜller M, Hoidis S, Smolders J (2008) A physiological frequencyposition map for the chinchilla cochlea. Association for Research in Otolaryngology Mid-winter Meeting Abstracts, 31:73-74

NARAYAN SS, Ruggero MA (2000) Basilar-membrane mechanics at the hook region of the chinchilla cochlea. In: Wada H, Takasaka T, Ikeda K, Ohyama K, Koike T (eds) Recent developments in auditory mechanics. World Scientific, Singapore, pp 95-101

Narayan SS, Temchin AN, Recio A, Ruggero MA (1998) Frequency tuning of basilar membrane and auditory nerve fibers in the same cochleae. Science 282:1882-1884

NutTall AL, Dolan DF (1993) Two-tone suppression of inner hair cell and basilar membrane responses in the guinea pig. J Acoust Soc Am 93:390-400

Ohlemiller KK, Echteler SM, Siegel JH (1991) Factors that influence rate-versus-intensity relations in single cochlear nerve fibers of the gerbil. J Acoust Soc Am 90:274-287

Palmer AR, Russell IJ (1986) Phase-locking in the cochlear nerve of the guinea-pig and its relation to the receptor potential of inner hair-cells. Hear Res 24:1-15

Palmer AR, Shackleton TM (2009) Variation in the phase of response to low-frequency pure tones in the guinea pig auditory nerve as functions of stimulus level and frequency. JARO 10:233-250

Pfeiffer RR, Kim DO (1975) Cochlear nerve fiber responses: distribution along the cochlear partition. J Acoust Soc Am 58:867-869 
PfeifFER RR, MolnaR CE (1970) Cochlear nerve fiber discharge patterns: relationship to the cochlear microphonic. Science 167:1614-1616

Recio-Spinoso A, Temchin AN, Van Dijk DP, Fan YH, Ruggero MA (2005) Wiener-kernel analysis of responses to noise of chinchilla auditory-nerve fibers. J Neurophysiol 93:3615-3634

Ren T, NutTall AL (2001) Basilar membrane vibration in the basal turn of the sensitive gerbil cochlea. Hear Res 151:48-60

RHoDE WS (1971) Observations of the vibration of the basilar membrane in squirrel monkeys using the Mössbauer technique. J Acoust Soc Am 49:1218-1231

RHodE WS (2007) Basilar membrane mechanics in the 6-9 KHz region of sensitive chinchilla cochleae. J Acoust Soc Am 121:2792-2804

RHode WS, COOPER NP (1996) Nonlinear mechanics in the apical turn of the chinchilla cochlea in vivo. Audit Neurosci 3:101-121

RHode WS, CoOper NP (1997) Nonlinear mechanisms in the apical turn of the chinchilla cochlea. In: Lewis ER, Long GR, Lyon RF, Narins PM, Steele CR, Hecht-Poinar E (eds) Diversity in auditory mechanics. World Scientific, Singapore, pp 318-324

RHode WS, Recio A (2000) Study of mechanical motions in the basal region of the chinchilla cochlea. J Acoust Soc Am 107:3317-3332

Robles L, Ruggero MA (2001) Mechanics of the mammalian cochlea. Physiol Rev 81:1305-1352

RONKEN DA (1986) Anomalous phase relations in threshold-level responses from gerbil auditory nerve fibers. J Acoust Soc Am $79: 417-425$

Rose JE, Hind JE, Anderson DJ, Brugge JF (1971) Some effects of stimulus intensity on response of auditory nerve fibers in the squirrel monkey. J Neurophysiol 34:685-699

Ruggero MA, Rich NC (1983) Chinchilla auditory-nerve responses to low-frequency tones. J Acoust Soc Am 73:2096-2108

Ruggero MA, Rich NC (1987) Timing of spike initiation in cochlear afferents: dependence on site of innervation. J Neurophysiol 58:379-403

Ruggero MA, Rich NC (1991) Furosemide alters organ of Corti mechanics: evidence for feedback of outer hair cells upon the basilar membrane. J Neurosci 11:1057-1067

Ruggero MA, Rich NC, Shivapuja BG, Temchin AN (1996) Auditorynerve responses to low-frequency tones: intensity dependence. Audit Neurosci 2:159-185

Ruggero MA, Rich NC, Recio A, Narayan SS, Robles L (1997) Basilarmembrane responses to tones at the base of the chinchilla cochlea. J Acoust Soc Am 101:2151-2163

Ruggero MA, Narayan SS, Temchin AN, Recio A (2000) Mechanical bases of frequency tuning and neural excitation at the base of the cochlea: comparison of basilar-membrane vibrations and auditory-nerve-fiber responses in chinchilla. Proc Natl Acad Sci U S A 97:11744-11750

Sachs MB, Aвbas PJ (1974) Rate versus level functions for auditorynerve fibers in cats: tone-burst stimuli. J Acoust Soc Am 56:18351847
Sachs MB, Winslow RL, Sokolowski BH (1989) A computational model for rate-level functions from cat auditory-nerve fibers. Hear Res 41:61-69

Sellick PM, Patuzzi R, Johnstone BM (1983) Comparison between the tuning properties of inner hair cells and basilar membrane motion. Hear Res 10:93-100

SEWELL WF (1984) The effects of furosemide on the endocochlear potential and auditory-nerve fiber tuning curves in cats. Hear Res 14:305-314

Temchin AN, Ruggero MA (2000) The phases of auditory-nerve fiber responses to tones: dependence on stimulus frequency and intensity. J Acoust Soc Am 107:2900

Temchin AN, Ruggero MA (2001) Parameters of the mechanical traveling wave in the chinchilla cochlea derived from auditorynerve fiber response phases. Assoc Res Otolaryngol, Midwinter Meet, Abstracts 24:156

Temchin AN, Rich NC, Ruggero MA (1997) Low-frequency suppression of auditory nerve responses to characteristic frequency tones. Hear Res 113:29-56

Temchin AN, Recio-Spinoso A, Van Dijk DP, Ruggero MA (2005) Wiener kernels of chinchilla auditory-nerve fibers: verification using responses to tones, clicks, and noise and comparison with basilar-membrane vibrations. J Neurophysiol 93:3635-3648

Temchin AN, Rich NC, Ruggero MA (2008A) Threshold tuning curves of chinchilla auditory nerve fibers. II. Dependence on spontaneous activity and relation to cochlear nonlinearity. J Neurophysiol 100:2899-2906

Temchin AN, Rich NC, Ruggero MA (2008в) Threshold tuning curves of chinchilla auditory-nerve fibers. I. Dependence on characteristic frequency and relation to the magnitudes of cochlear vibrations. J Neurophysiol 100:2889-2898

Temchin AN, Rich NC, Ruggero MA (2009) Threshold tuning curves of chinchilla auditory-nerve fibers predict cochlear phasefrequency curves and impulse-response frequency glides. Association for Research in Otolaryngology Mid-winter Meeting Abstracts 32:209

Van Der Heijden M, Joris PX (2003) Cochlear phase and amplitude retrieved from the auditory nerve at arbitrary frequencies. J Neurosci 23:9194-9198

Van Der Heijden M, Joris PX (2006) Panoramic measurements of the apex of the cochlea. J Neurosci 26:11462-11473

WeIss TF, Rose C (1988) A comparison of synchronization filters in different auditory receptor organs. Hear Res 33:175-179

Zagaeski M, Cody AR, Russell IJ, Mountain DC (1994) Transfer characteristic of the inner hair cell synapse: steady-state analysis. J Acoust Soc Am 95:3430-3434

Zinn C, Maier H, Zenner H, Gummer AW (2000) Evidence for active, nonlinear, negative feedback in the vibration response of the apical region of the in-vivo guinea-pig cochlea. Hear Res 142:159-183 